\title{
Coherence of Seismic Body Waves From Local Events as Measured by a Small-Aperture Array
}

\author{
Frank L. Vernon, ${ }^{1}$ Jon Fletcher, ${ }^{2}$ Linda Carroll,${ }^{2}$ Alan Chave, ${ }^{3}$ and Eugene Sembera ${ }^{2}$
}

\begin{abstract}
Eight local earthquakes were recorded during the operation of a small-aperture seismic array at Pinyon Flat, California. The site was chosen for its homogeneous granitic geology and its planar topography. Amplitude spectral ratios for the same signal measured at different stations had average values of less than 2 and maximum values of 7. Magnitude-squared coherences were estimated for all station pairs. These estimates were highest for the $P$ wave arrivals on the vertical component and lowest for the $P$ wave recorded on the transverse component. At $500 \mathrm{~m}$ station separation the $P$ and $S$ waves were incoherent above $15 \mathrm{~Hz}$ and $10 \mathrm{~Hz}$, respectively. Coherence for both the $P$ and $S$ waves decrease as frequency increases and as distance increases. The coherence of signals from borehole sensors located at 300 and $150 \mathrm{~m}$ depth displays higher average coherence than equally spaced sites located on the surface. The results here suggest that even for sites that appear to be very similar, that is, those which are located on a planar surface within a few meters of granite bedrock, the measured seismic wavefield can be distorted substantially over scale lengths of $500 \mathrm{~m}$. Coherence properties were calculated from synthetic seismograms which were computed for velocity models with exponential and self similar distribution perturbations. Standard deviations of $10 \%$ are not sufficient for the random velocity distributions to approximate the results from the small-aperture array.
\end{abstract}

\section{INTRODUCTION}

It is assumed for measurements of local earthquakes with small source dimensions that the seismic energy is coherent near the source for small perturbations of takeoff angles. As the signals propagate through the Earth they become less coherent due to scattering, but the coherent frequency bands should contain significant information about the seismic source. In recent years there has been considerable interest in the variation of seismic waveforms measured at inter-station spacings of tens to hundreds of meters. Several studies have demonstrated considerable variability in the seismic wavefield over these short distances. The response of shallow sediment-filled valleys was carefully measured by King and Tucker [1984] and Tucker and King [1984], who placed an array with this range of station spacings across the Chusal and Yasman valleys in the Soviet Union. Tucker et al. [1984] examined the differences between stations located on hard rock which included ridge, slope, and tunnel sites. McLaughlin et al. [1983] investigated the wavenumber spectra from, and coherence between, stations in a small array that recorded a nearby nuclear explosion. This array was located on Pahute Mesa in the Nevada Test Site at a level location but in a complex geological setting. There was significant incoherent energy above $6 \mathrm{~Hz}$ for $P$ waves and above $4 \mathrm{~Hz}$ for $S$ waves. They found that the correlations between stations is higher in the direction transverse to propagation.

In this paper, results are presented from a study of the variability of seismic spectra and the spatial coherence of the seismic wavefields over horizontal distances of $50-500 \mathrm{~m}$. These data were recorded on a nine-station seismic array operated on a fairly level granitic terrain for eight months in 1985 . Eight events,

\footnotetext{
${ }^{1}$ Institute of Geophysics and Planetary Physics, Scripps Institution of Oceanography, University of California, San Diego, La Jolla.

${ }^{2}$ U.S. Geological Survey, Menlo Park, California.

${ }^{3}$ AT\&T Bell Laboratories, Murray Hill, New Jersey.
}

Copyright 1991 by the American Geophysical Union.

Paper number 91JB00193.

0148-0227-91JB/91/00193 \$05.00 which have epicentral distances between 14 and $40 \mathrm{~km}$ distributed with at least one in every quadrant of azimuth, were analyzed. The criteria for choosing the array site are discussed in section 2 . The experimental design and the events recorded by this array are summarized in sections 3 and 4, respectively. The data analysis procedures, which include multitaper spectral and coherence estimates, are presented in section 5. Examples of analysis results are shown in section 6. section 7 examines a set of borehole recordings and Section 8 presents modeling results. Section 9 contains a more detailed discussion, and section 10 lists the conclusions. The appendix contains the mathematical details of the multitaper spectral analysis procedures which differ only slightly from the original version given in a seminal paper by Thomson [1982].

\section{Site CHOICE}

Site selection for this seismometer array is based on several criteria. First, the array must be located on a hard rock site that appears to be geologically uniform where the seismic signals are not expected to be distorted by geological structures near the array. Second, the region of the site must have little topographic variation which can possibly amplify or attenuate seismic signals [Geli et al., 1988]. Finally, the site should be in an active seismic region.

Pinyon Flat, which is located in the northernmost part of the Peninsular Ranges batholith in southern California, is a site that satisfies these conditions. The site is situated on the northern part of the Santa Rosa Mountain Pluton (also known as Haystack Pluton) [Dibblee, 1981]. This pluton has a granodiorite composition [Parcel, 1981] and is approximately $30 \mathrm{~km}$ long and $15 \mathrm{~km}$ wide in a NW-SE orientation.

The array was installed at the Piñon Flat Observatory (PFO) at an elevation of $1280 \mathrm{~m}$ above sea level. Figure 1 [from Wyatt, 1982] shows a topographic map of the area with the array located inside the heavy black ring. Pinyon Flat is bounded on the north by Asbestos Mountain (elevation $1585 \mathrm{~m}$ ) and on the south by the Santa Rosa Mountains. Two canyons, Palm Canyon and Deep Canyon, bound Pinyon Flat on the west and east, respectively. The general array area has little topographic relief within several kilometers of the array. 

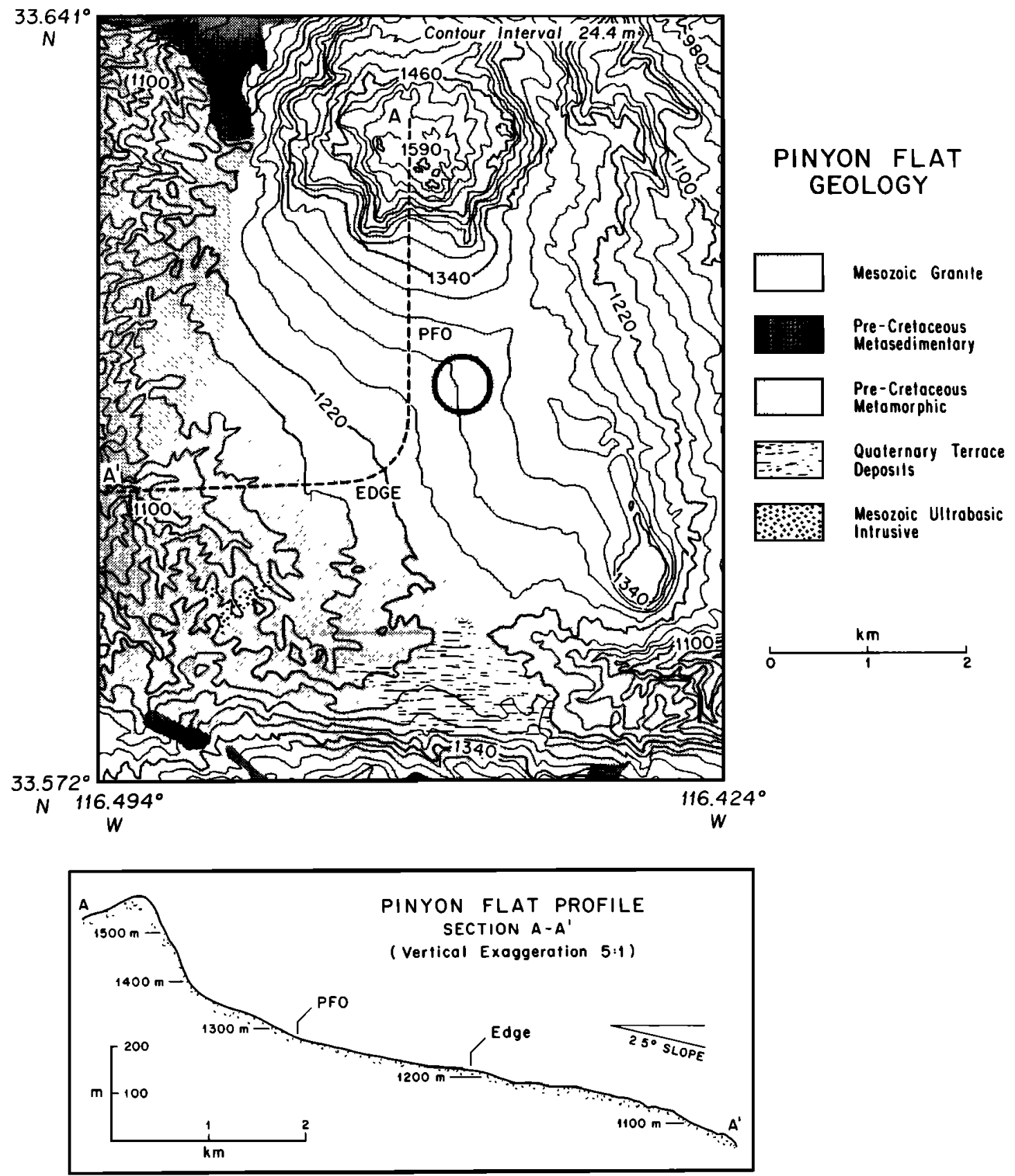

Fig. 1. Geology and topography of Pinyon Flat from Wyatt [1982]. The heavy circle shows the radius of this seismic array.

The near-surface geology of the PFO site has been studied in some detail. Wyatt [1982] found the top $1 \mathrm{~m}$ of ground to be almost totally decomposed granodiorite. This grades into grus, then corestones, and becomes jointed granodiorite at a depth of 25 $\mathrm{m}$ or less. In the same study, results of a shallow seismic refraction survey at PFO were presented. This survey showed that the compressional velocities were confined by the extremal bounds to $2.0 \mathrm{~km} \mathrm{~s}^{-1}$ to $3.3 \mathrm{~km} \mathrm{~s}^{-1}$ at $18 \mathrm{~m}$ depth. $P$-wave logging of a 300-m borehole was conducted by Fletcher et al. [1990]. They determined compressional velocities of $4 \mathrm{~km} \mathrm{~s}^{-1}$ at $65 \mathrm{~m}$, rising to over $5 \mathrm{~km} \mathrm{~s}^{-1}$ by $80 \mathrm{~m}$ depth. These measurements suggest that this site has a thin weathered layer overlying a rigid basement.

The seismicity in the region is quite high since the array is located about $12 \mathrm{~km}$ northeast of the San Jacinto fault zone and about $25 \mathrm{~km}$ southwest of the San Andreas fault zone [Wyatt,
1982]. The seismicity of magnitude $M_{L} \geq 3$ events in the years 1980-1984 is shown in Figure 2, along with the major fault traces.

\section{EXPERIMENTAL DESIGN}

For this experiment it was important to ensure that the transfer functions of the sensors and the recording instruments at the sites were very nearly identical so that the differences between the recorded signals were caused solely by the differences in ground motion at each recording site. To accomplish this result the design, construction, and equipment setup at all the individual recording sites were as identical as possible.

The array consisted of nine stations. Each site included a triaxial set of Mark Products L-22 seismometers. The outputs of these 


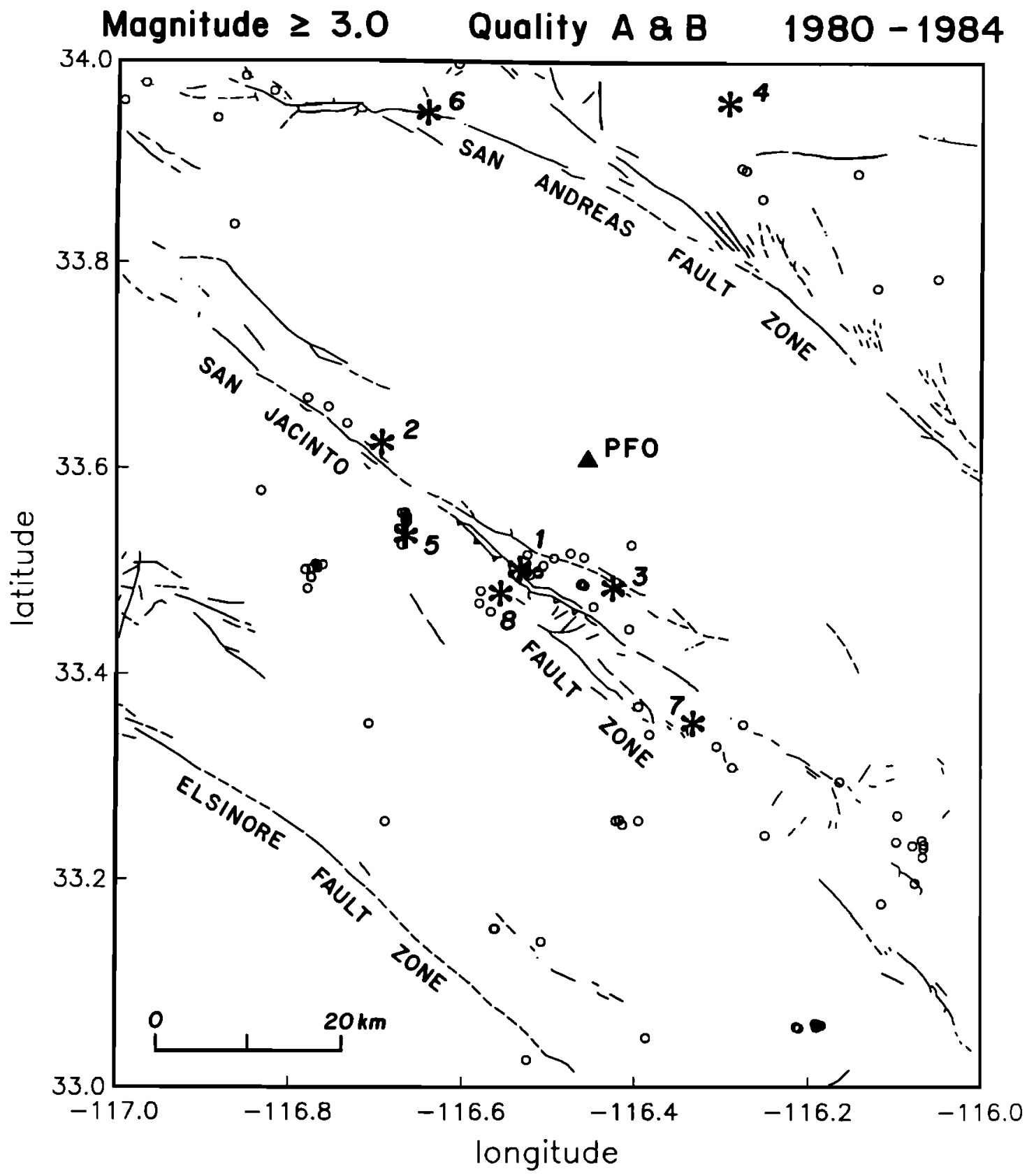

Fig. 2. Location of magnitude 3 or greater events (circles) from the Caltech catalog for the years 1980-1984. The events which were recorded on the small aperture array are marked with asterisks.

2-Hz seismometers were recorded by Sprengnether DR-100 digital cassette recorders. Each channel was recorded with a 12-bit analog-to-digital converter at 200 samples/s. The antialiasing filters had a five-pole Butterworth response with a $50-\mathrm{Hz}$ corner frequency.

The array was set up as a set of three nested equilateral triangles (Figure 3) in February 1985. The center point of the array was located on top of the southeast vault of the NW-SE laser strainmeter at PFO. Each sensor was precisely located using a Nikon NTD-2 infrared electronic distance measuring device. The first station sited was AZG while the other $300-\mathrm{m}$ radius stations (AZH and AZI) were located at clockwise angles of $120^{\circ}$ and $240^{\circ}$, respectively, from the reference line of the center point to AZG. The middle triangle of stations AZD, AZE, and AZF were located at a distance of $100 \mathrm{~m}$ from the center point and $60^{\circ}, 180^{\circ}$, and $300^{\circ}$ from the reference line. The inner triangle stations $\mathrm{AZA}, \mathrm{AZB}$, and $\mathrm{AZC}$ were placed at a radius of $30 \mathrm{~m}$ and at $30^{\circ}$, $150^{\circ}$, and $270^{\circ}$ angles from the reference. After initial installation, station AZG was subsequently moved to site AZJ to eliminate $60 \mathrm{~Hz}$ power line noise. The site AZJ was also $300 \mathrm{~m}$ away from the center point, but at a clockwise angle of $21^{\circ}$ from the AZG site.

Each station was battery powered. The digital recorders were modified to run off a single external $1 \mathrm{MHz}$ clock to synchronize the taking of each data sample across the array. Each digitizer operated with its own independent trigger. The equipment was serviced at intervals of 10 to 14 days. After the data were recorded in the field the cassettes were returned to the U.S. Geological Survey (USGS) labs at Menlo Park and played back onto a computer. Each event from each digitizer was plotted and 


\section{Coherence Array Layout}

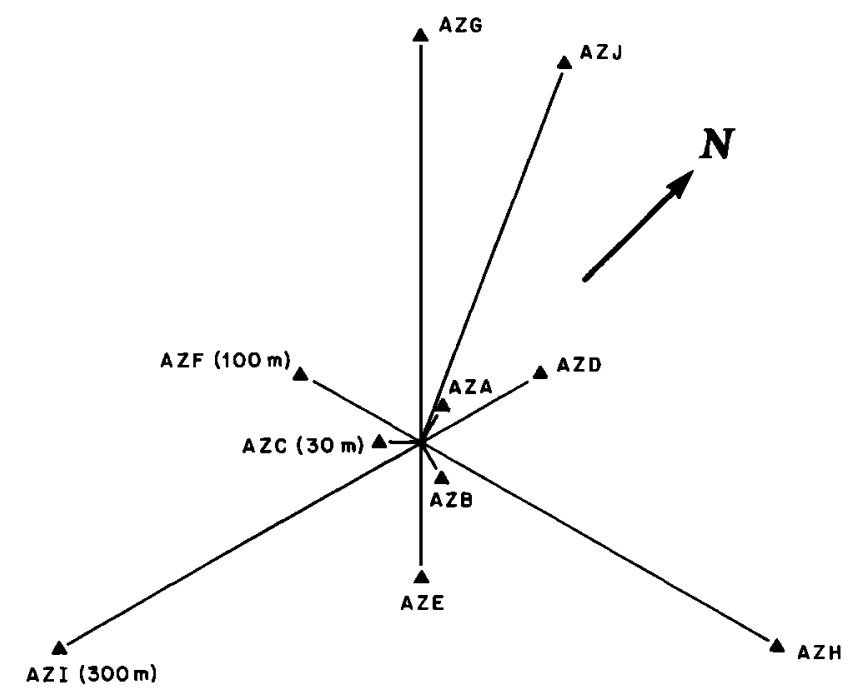

Fig. 3. Layout of coherence array.

checked for recording problems or false triggers. Events that were recorded on five or more stations were chosen for detailed analysis.

\section{The Data Set}

During the operation of this array from late January to September 1985 there were eight events that triggered five or more recorders simultaneously. These events had local magnitudes $M_{L}$ between 2.6 and 3.9, as estimated from the southern California seismic network array data [Norris et al., 1986; Given et al., 1987]. The events, marked with asterisks in Figure 2, have epicentral distances ranging from 14 to $42 \mathrm{~km}$ and depths between 4 and $16 \mathrm{~km}$. None of these events were in the same place, and there were seven independent azimuths for this set of earthquakes. Table 1 shows various source parameters including locations, epicentral distance and azimuth from PFO, local magnitude, seismic moment, and stress drop. The moment and stress drop estimates were calculated from data collected on the Anza seismic array using the method described by Fletcher et al. [1987].

An example of a seismic time series from this experiment is shown in Figure 4. The figure displays the transverse components of event 8 , located $17 \mathrm{~km}$ southwest of the array. Note that the visual correlation between each series is apparently quite low for all parts of the seismogram except for the large low-frequency pulse at the beginning of the shear wave arrival. In general, the seismograms for the three components for a given event show some similarity to each other during the $P$ and $S$ phases while the coda for each phase appears to be random in character.

\section{Data Analysis Techniques}

Since we are dealing with short time series and have all the attendant problems of spectral leakage and variability for the estimates, we decided to use the multiple-taper spectral analysis technique devised by Thomson [1982]. The application of this technique for calculation of spectra from seismic data has been described by Park et al. [1987]. We used a slightly modified form for the calculation of the spectra described here. A derivation of these modified equations is given in the appendix. In practice, spectra calculated by both methods from the same data are similar in appearance.

The multitaper power spectral method attempts to minimize the bias due to spectral leakage outside a chosen bandwidth. The implicit assumptions for this technique are that the data are a Gaussian distributed, evenly sampled, unaliased, ergodic, stationary time series. A set of tapers $\left\{v_{t}^{(k)}(N, W)\right\}_{k=0}^{K-1}$, are generated which are functions of the time series length $N$ and the specified "inner" bandwidth $W$. The tapers are the set of eigenvectors [Park et al., 1987] with associated eigenvalues $1>\lambda_{k}(N, W)>0$. The data $x_{t}$ are multiplied by each of the $K$ tapers in turn, and the discrete Fourier transform is calculated,

$$
y_{k}(f)=\sum_{t=0}^{N-1} v_{t}^{(k)}(N, W) x_{t} e^{-i 2 \pi f t} .
$$

An adaptive spectral estimate $S(f)$ is a weighted average of the $K y_{k}(f)$ values. The weights $d_{k}(f)$ depend on frequency and on the taper order $k$, since the bias resistance for each taper

$$
S(f)=\frac{A}{K} \frac{\sum_{k=0}^{K-1} \lambda_{k} d_{k}^{2}(f)\left|y_{k}(f)\right|^{2}}{\sum_{k=0}^{K-1} d_{k}^{2}(f)}
$$

decreases as $k$ increases. The coefficient

$$
A=\sum_{k=0}^{K-1} \lambda_{k}^{-1}
$$

and the weights $d_{k}(f)$ are calculated using the recursion relation in the appendix.

As pointed out by Thomson [1982], the orthogonal properties of the multitaper spectral analysis can be extended to calculate coherences. If there are two series $x_{t}^{i}$ and $x_{t}^{j}$ which have equally sampled data, then (2) can be generalized to

TABLE 1. Source Parameters

\begin{tabular}{ccccccccccr}
\hline Event & $\begin{array}{c}\text { Date, } \\
\text { 1985 }\end{array}$ & $\begin{array}{c}\text { Time, } \\
\text { UT }\end{array}$ & $\begin{array}{c}\text { Latitude, } \\
{ }^{\circ} \mathrm{N}\end{array}$ & $\begin{array}{c}\text { Longitude, } \\
{ }^{W}\end{array}$ & $\begin{array}{c}\text { Depth, } \\
\text { km }\end{array}$ & $\begin{array}{c}\text { Distance, } \\
\text { km }\end{array}$ & $\begin{array}{c}\text { Azimuth, } \\
\text { deg }\end{array}$ & ML & $\begin{array}{c}\text { MOM, } \\
\text { dyne-cm }\end{array}$ & $\begin{array}{r}\text { SD, } \\
\text { bars }\end{array}$ \\
\hline 1 & Jan 27 & 0158 & 33.502 & 116.534 & 12.9 & 14.0 & 212 & 2.8 & $6.5 \times 10^{19}$ & 5.0 \\
2 & Jan 27 & 2003 & 33.628 & 116.695 & 16.1 & 22.3 & 275 & 2.6 & $1.1 \times 10^{20}$ & 10.6 \\
3 & Feb 21 & 0754 & 33.483 & 116.426 & 11.5 & 14.3 & 169 & 3.1 & $8.2 \times 10^{19}$ & 11.9 \\
4 & Feb 28 & 0442 & 33.960 & 116.293 & 10.1 & 42.0 & 21 & 3.7 & - & - \\
5 & Apr 08 & 1613 & 33.537 & 116.670 & 11.5 & 21.4 & 248 & 3.0 & $1.4 \times 10^{20}$ & 8.0 \\
6 & May 25 & 1550 & 33.949 & 116.643 & 5.1 & 41.6 & 335 & 3.2 & $1.9 \times 10^{20}$ & 15.4 \\
7 & Jun 05 & 1810 & 33.353 & 116.333 & 4.11 & 30.7 & 158 & 3.9 & $8.8 \times 10^{20}$ & 9.1 \\
8 & Jun 29 & 1823 & 33.479 & 116.556 & 13.5 & 17.2 & 213 & 3.2 & $3.0 \times 10^{20}$ & 33.6 \\
\hline
\end{tabular}

ML is local magnitude, MOM is seismic moment and SD is stress drop. 
Transverse Component Event 8

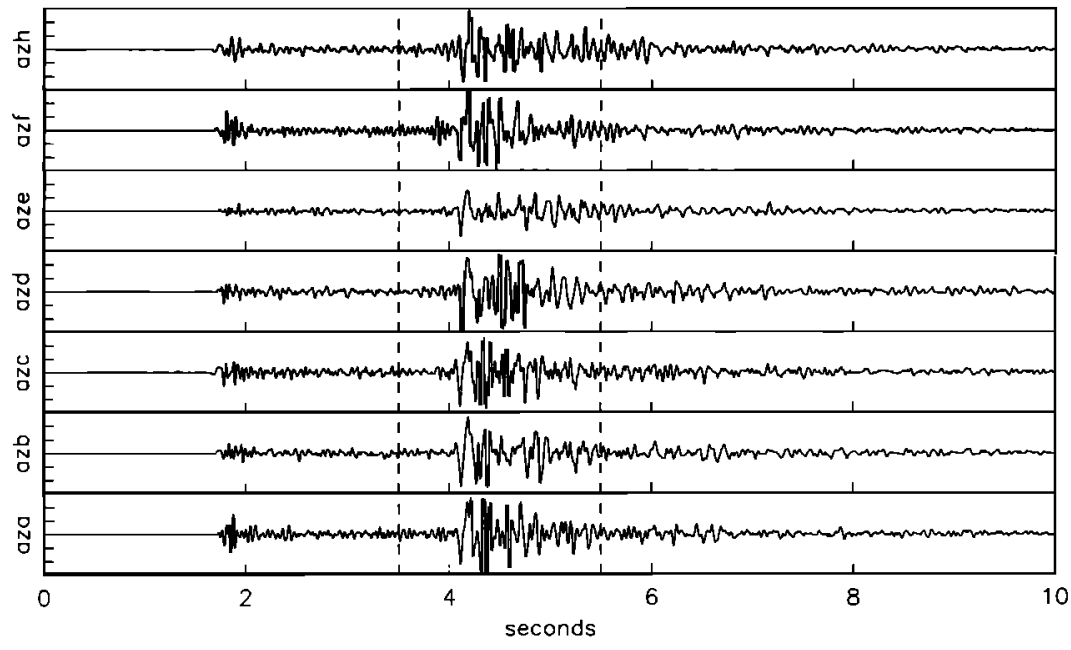

Fig. 4. Seismograms recorded on the transverse component from event 8 . The section of the seismograms used for the shear wave analysis is bounded by the dashed lines.

$$
S_{l j}(f)=\frac{A}{K} \frac{\sum_{k=0}^{K-1} \lambda_{k} d_{k}^{l}(f)\left(y_{k}^{l}(f)\right)^{*} d_{k}^{\prime}(f)\left(y_{k}^{\prime}(f)\right)}{\left[\sum_{k=0}^{K-1} d_{k}^{l}(f)^{2}\right]^{1 / 2}\left[\sum_{k=0}^{K-1} d_{k}^{\prime}(f)^{2}\right]^{1 / 2}}
$$

If $i=j$, then (3) is the autospectrum (2). If $i \neq j$, then $S_{i j}(f)$ is the cross spectrum between $x_{t}^{t}$ and $x_{t}^{j}$, where the weights $d_{k}^{l}(f)$ are the same ones used for the $S_{j j}(f)$. The magnitude squared coherence $\gamma_{l}^{2}(f)$ is then found directly from

$$
\gamma_{i j}^{2}(f)=\frac{\left|S_{i j}(f)\right|^{2}}{S_{u}(f) S_{j J}(f)}
$$

and the phase $\phi(f)$ is just the phase of $S_{u}(f)$. Multitaper coherence estimation has been used by Lanzerotti et al. [1986], where they choose $d \xi(f) \approx 1$. Here we have a larger dynamic range, so it is necessary to use the $d_{k}(f)$ explicitly.

One property of the multitaper technique is the orthogonality and hence independence of each tapered estimate in both the frequency and time domains. The independence of the $y_{k}(f)$ implies that estimates of the mean, bias, and variance for the power spectra and the magnitude-squared coherence can be made. Unfortunately, the underlying probability distribution is not always known precisely enough for $S(f)$ and $\gamma^{2}(f)$ with real data. Furthermore, there are always a limited number of samples for the estimation of $S(f)$ and $\gamma^{2}(f)$. Under these constraining conditions, with an unknown probability distribution and the small number of samples, it is necessary to use an empirical method of estimating bias and variance. Lanzerotti et al. [1986] first introduced the use of jackknife statistics with multitaper estimates to evaluate the variance of the pseudoautocorrelations of magnetic field data and cable voltages. Lindberg and Park [1987] also used a jackknifed multitaper estimate for the errors in peak frequency measurements of the Earth's normal modes.

The development and background of the jackknife are presented by Miller [1974] and Efron [1982], with an outline of the pertinent equations included here. The basic jackknife starts with the assumption that there are $n$ independent and identically distributed random variables $Z_{l}(1 \leq \ell \leq n)$ from an unknown probability distribution. For each random variable $Z_{l}$ there corresponds an observation of that variable, $z_{\ell}$. The quantity of interest is estimated by the statistic $\hat{\theta}=\hat{\theta}\left(z_{1}, z_{2}, \ldots, z_{n}\right)$. The jackknife makes use of a set of deleted estimates $\hat{\theta}_{(t)}$ where the $i$ th observation $z_{l}$ is not used in the estimate:

$$
\hat{\theta}_{(t)}=\hat{\theta}\left(z_{1}, z_{2}, \ldots, z_{t-1}, z_{l+1}, \ldots, z_{n}\right)
$$

The jackknifed estimate of the mean of $\hat{\theta}$ is

$$
\hat{\boldsymbol{\theta}}_{(\cdot)}=\frac{1}{n} \sum_{i=1}^{n} \hat{\boldsymbol{\theta}}_{(i)}
$$

Tukey [1958] showed that an estimate of the variance of $\hat{\theta}$ is

$$
\operatorname{var}\{\hat{\theta}\}=\frac{n-1}{n} \sum_{i=1}^{n}\left(\hat{\theta}_{(t)}-\hat{\theta}_{(\cdot)}\right)^{2}
$$

Miller [1974] points out that it is beneficial, if possible, to transform the statistic $\hat{\theta}$ so that its underlying probability distribution becomes more Gaussian. This can be important when estimating standard errors on quantities such as variance, which has a range of $[0, \infty)$, or magnitude squared coherence, which has a range of $[0,1]$. The transformations used to convert to a more normal distribution for power spectra are $\theta(f)=\log (S(f))$ [Miller, 1968]; and for magnitude-squared coherence, $\boldsymbol{\theta}(f)=\operatorname{arctanh}\left(\sqrt{\gamma^{2}(f)}\right)$ [Jenkins and Watts, 1968].

To create the $K$-deleted estimates $\theta$, with $i=1, \ldots, K$ to be used for the estimation of power spectra variance, (2) is modified to read

$$
S_{1}(f)=\frac{A-\lambda_{l}^{-1}}{K-1} \frac{\sum_{k=0, k \neq i}^{K-1} \lambda_{k} d_{k}^{2}(f)\left|y_{k}(f)\right|^{2}}{\sum_{k=0, k \neq 1}^{K-1} d_{k}^{2}(f)}
$$

Equations (3) and (4) are modified in a similar manner to determine the deleted estimate of magnitude squared coherence.

\section{DATA ANALYSIS}

The analysis of the body wave phases for each event starts with the rotation of the time series into radial and tangential components followed by calculating the power spectrum of each component. For this data set a time-bandwidth product $(\mathrm{NW})$ of 6.5 with 12 tapers was used in each computation. This gives at most 
24 degrees of freedom for $S(f)$ at each $f$. The time series have been adjusted in time by synchronizing to the $P$ wave arrivals. Each spectrum used $2 \mathrm{~s}$ of the time series centered on the particular phase of interest, as shown in Figure 4. Next, the spectra from each station were combined to estimate an average of the log spectrum, $S_{A}(f)$, using the following equation:

$$
S_{A}(f)=\exp \left[\frac{1}{N} \sum_{t=1}^{N} \ln \left(S_{t}(f)\right)\right]
$$

where $S_{l}(f)$ is the spectrum at station $i$ and $N$ is the total number of stations. The $95 \%$ confidence limits $C_{ \pm}(f)$ are found assuming Gaussian distributed variates, yielding

$$
C_{ \pm}(f)=\alpha^{ \pm 1}(f) S_{A}(f)
$$

where

$$
\alpha^{ \pm 1}(f)=\exp \left[2 \sqrt{\operatorname{VAR}\left(\ln \left(S_{i}(f)\right)\right)}\right]
$$

and $\operatorname{VAR}(\beta)$ is the variance of $\beta$.

The gross characteristics of the averaged log spectra are all quite similar (Figure 5) to those of the individual spectra. The averaged $\log$ spectrum $S_{A}(f)$ from the $P$ wave recorded on the vertical component for event 1 is shown in Figure 5a. Because the original time series are recordings of ground velocity, the spectra decrease in power as frequency approaches $0 \mathrm{~Hz}$ as
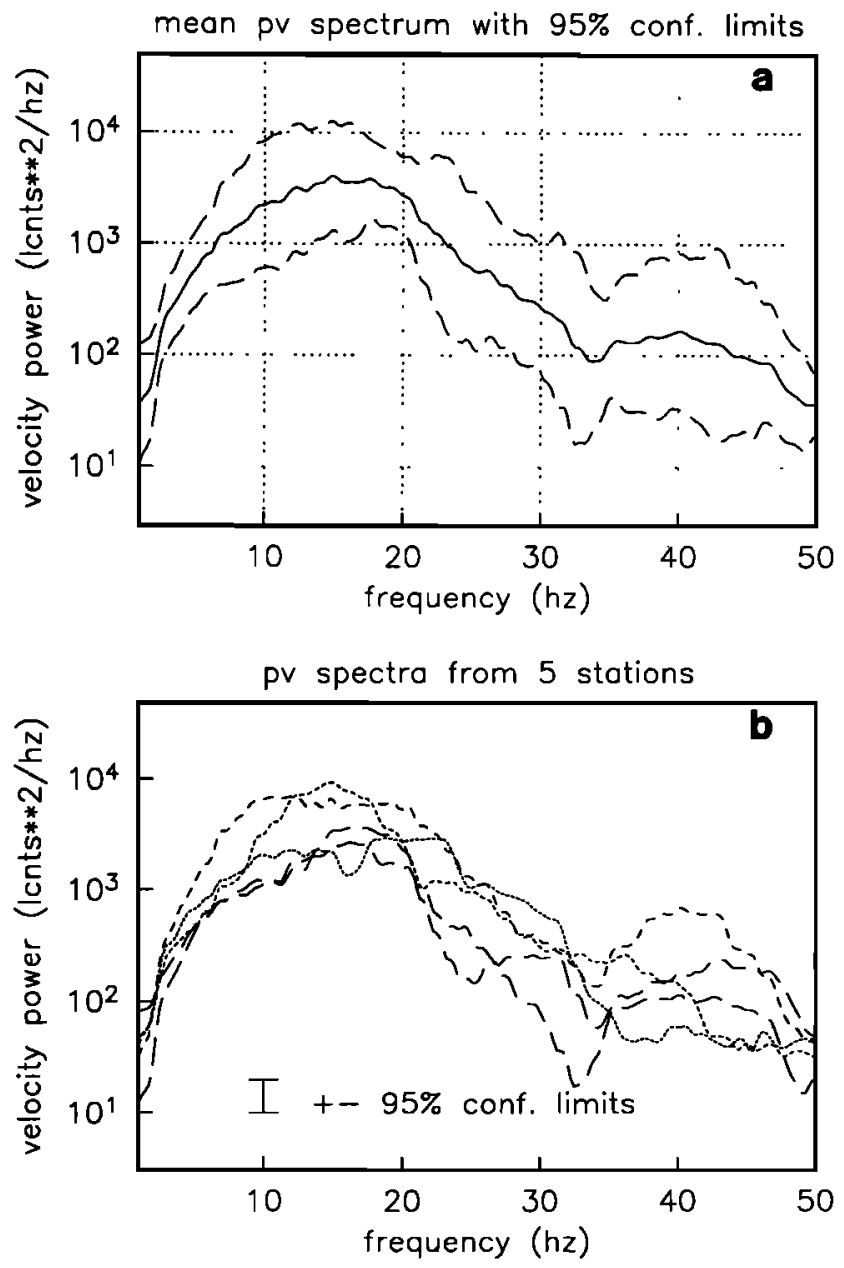

Fig. 5. (a) Mean log power spectrum with $95 \%$ confidence limits for the $P$ wave on the vertical components of event 1 . (b) Individual spectra from which the log average spectrum was calculated. Average error bars for individual spectra are given. predicted by $\omega^{-2}$ or Brune's model and confirmed by observation. The velocity power peak for all phases is found to be between 5 and $22 \mathrm{~Hz}$.

A remarkable feature of the averaged log spectra is their variance. The $5-95 \%$ confidence limits $\alpha_{ \pm}(f)$, determined from the variance of the log spectra between stations, has a mean value of approximately 4 but can vary from 1.4 to over 13.0 as a function of frequency. For reference, the ratio of the 5-95\% quantities of a $\chi_{24}^{2}$ distribution is 2.83 . This comparison suggests that the variance probably is caused by an unmodeled process and is not caüsed by noise in the measurements. On closer examination of individual spectra, they can be divided into low- and highfrequency parts by their variance. The individual spectra used to form the averaged log spectra in Figure $5 a$ are shown in Figure $5 b$. From 0 to $15 \mathrm{~Hz}$ all the spectra are similar, differing by a scale factor. The detailed structures of the spectra are not correlated in the band above $15 \mathrm{~Hz}$ for these $P$ waves. The $S$ waves display similar phenomena except that decorrelation starts at approximately $10 \mathrm{~Hz}$. This feature cannot be explained by the error estimates of the individual spectra indicating that different processes are at work at different sites. The average double-sided 95\% confidence limit for each spectrum is shown on the plot and is much smaller than the differences between spectra. Clearly the details of the seismic body wave spectra above $15 \mathrm{~Hz}$ for $P$ waves and above $10 \mathrm{~Hz}$ for $S$ waves are controlled by variable localized site effects even at these short station spacings of less than $500 \mathrm{~m}$.

The second part of the analysis is an examination of the coherences between each station pair for every event. The coherences were calculated using data aligned to maximize the cross correlation between waveforms, and using the same spectral parameters ( $2 \mathrm{~s}$ of data, 12 tapers, time-bandwidth product $N W=6.5$ ) as in the power spectrum estimation. Figure 6 displays an example of the magnitude squared coherence $\left(\gamma^{2}\right)$ estimates from the transverse component of the shear wave time series in Figure 4. These coherence estimates use station AZA as the trace to which all other stations are compared. The coherence calculations were repeated with a different set of spectral parameters $(0.5 \mathrm{~s}$ of data, 6 tapers, $N W=3.5$ ) which did not produce significantly different results.

The estimates of $\gamma^{2}$ shown in Figure 6 gives the general characteristics of all the coherence estimates which were calculated. Each estimate has large random variations superimposed on a general trend of high $\gamma^{2}$ at low frequency decaying toward zero as the frequency increases. The highest $\gamma^{2}$ estimates averaged over frequency are found for the $P$ waves measured on the vertical components where $\gamma^{2}$ values of greater than 0.7 can be found out to over $40 \mathrm{~Hz}$. The detailed structure of the magnitude-squared coherence does not correlate from station to station on the same event (Figure 6), nor from event to event for fixed station pairs. Figure 7 shows the coherence estimates from a different event using the same master trace AZA. Notice the difference between coherences measured on the same station pairs in Figures 6 and 7. If the seismic wavefield is coherent near the source, then the difference between these observations must be caused by path effects. The variability of these estimates makes it difficult to characterize expected $\gamma^{2}$ properties using only single sets of station pairs or data from just one event.

The process of finding a general set of properties using all the $\gamma^{2}$ estimates for each component of the $P$ and $S$ waves (115 and 140 estimates, respectively) starts by examining the signal-tonoise properties of the power spectra. If the instrumental or ground noise is a significant fraction of the measured signal, then estimates of the signal coherence between two points will be 
Mag. Sq. Coherence $\mathrm{S}$ wave Transverse component Event 8
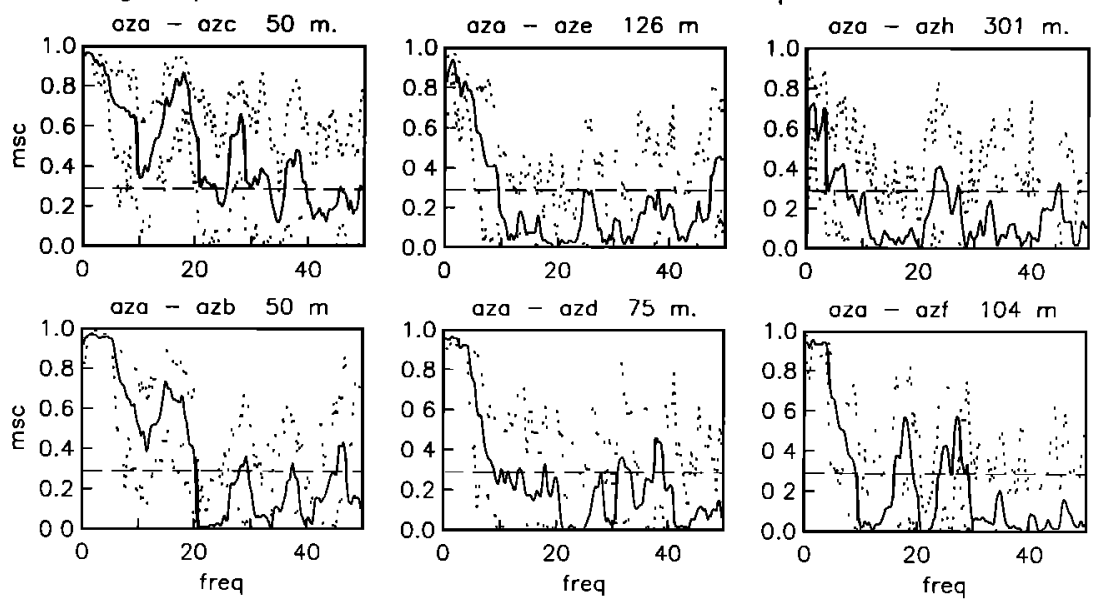

Fig. 6. The data from station AZA are the common time series and the $\gamma^{2}$ estimates of the common series (solid curve) with each of the other stations' data are shown in each successive plot for event 8 . The $\pm 95 \%$ confidence limits (short dashed curves) of the $\gamma^{2}(f)$ are plotted as well as the theoretical $95 \%$ confidence limit (for Gaussian data) that $\gamma^{2} \neq 0$ (longer dashed curves).
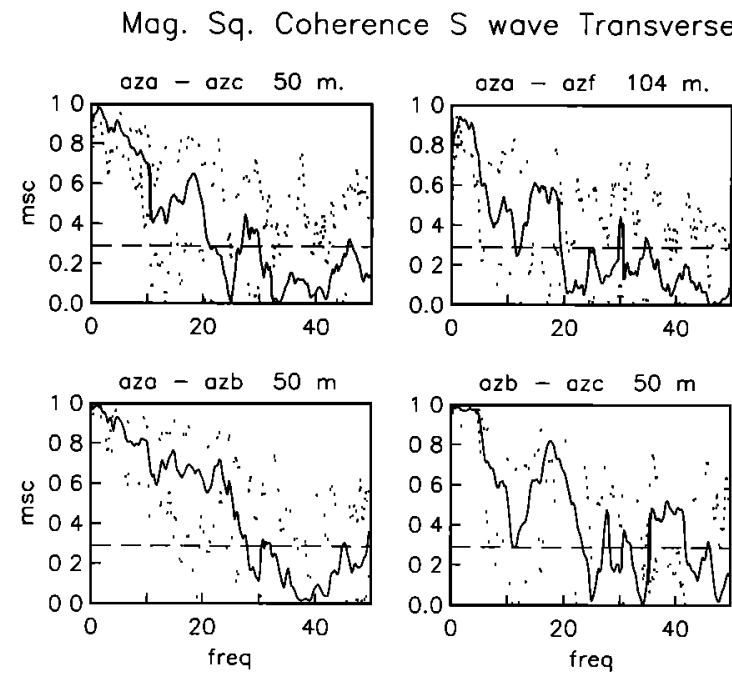

component Event 1
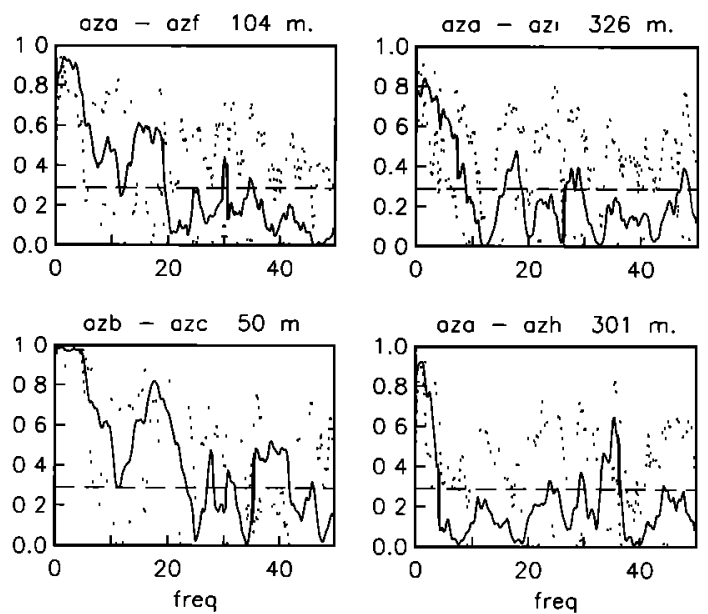

Fig. 7. The data from station AZA are the common time series and the $\gamma^{2}$ estimates of the common series with each of the other stations' data are shown in each successive plot for event 8 . Same conventions used as in Figure 6.

biased low. For each event in this data set the signal to noise ratio is similar for each seismogram. Wyatt and Berger [1980] investigated this problem of noise bias when they measured the coherence between the outputs of two adjacent tiltmeters. They assumed that the output of each instrument $x_{1}^{1}$ consisted of a common mode signal $u_{t}$ with an added noise signal $v_{t}^{\prime}$. If the power signal-to-noise ratio is defined by

$$
\sigma^{2}(f) \equiv \frac{U(f)}{V(f)}
$$

where $U(f)$ and $V(f)$ are the power spectra of the common signal and the independent noise, respectively, then the magnitude squared coherence can be estimated by

$$
\gamma_{l}^{2}(f)=\frac{1}{\left[1+\frac{1}{\sigma_{l}^{2}(f)}\right]\left[1+\frac{1}{\sigma_{J}^{2}(f)}\right]}
$$

where the subscripts $i$ and $j$ represent different sensors. If $\sigma_{\imath}^{2}=\sigma_{J}^{2}=20$, then $\gamma_{t j}^{2}=0.907$ by (9). In our experiment we approximate

$$
\sigma_{\imath}^{2}(f) \approx \frac{S_{l}(f)}{N_{\imath}(f)}
$$

where $S_{i}(f)$ and $N_{i}(f)$ are the power spectra of the signal of interest and the pre-event noise, respectively, and exclude any coherence estimates from further processing when $\sigma^{2}(f)<20$.

The estimates of $\gamma_{1}^{2}\left(f_{0}\right)$ were examined for all station pairs at $2.5 \mathrm{~Hz}$ increments over the range $2.5 \mathrm{~Hz} \leq f_{0} \leq 50 \mathrm{~Hz}$. For each frequency $f_{0}$, all estimates $\gamma_{t j}^{2}\left(f_{0}\right)$ for all events of a particular body wave and component were collected, converted to a more normal distribution ( $\left.\operatorname{arctanh} \sqrt{\gamma_{1 j}^{2}\left(f_{0}\right)}\right)$, and sorted by distance between measuring points. All estimates for the same distance were averaged. A weighted cubic spline fit to these average estimates of $\gamma_{l j}^{2}\left(f_{0}\right)$ was calculated as a function of distance. The results of the spline fits were combined for all $f_{0}$. These are presented in contour plots for the three components of the $P$ wave (Figure 8) and the $S$ wave (Figure 9). These plots give an average value of $\gamma^{2}$ as a function of frequency and distance. Values for which $\gamma^{2}(f, d)<0.3$ are not contoured because they are not significantly different from zero at the $95 \%$ confidence level. 


\section{COMPRESSIONAL WAVES}

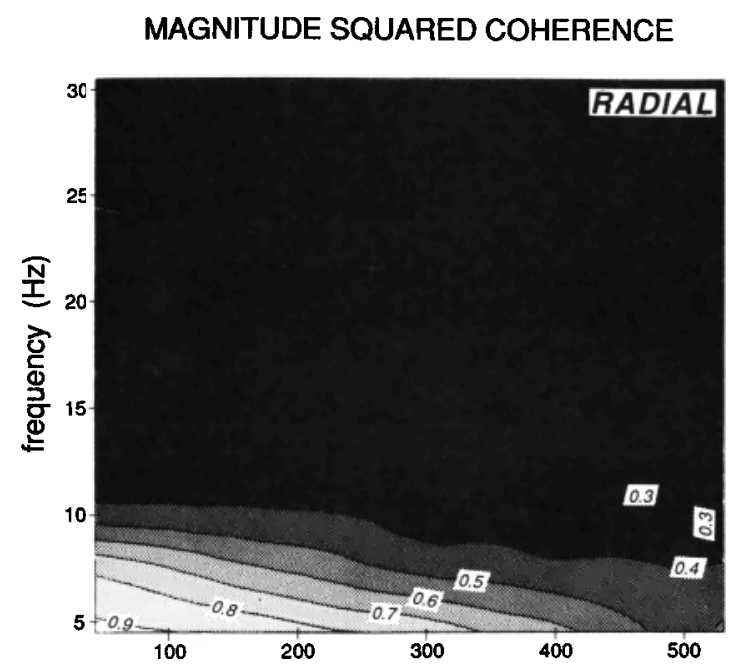

SIGNAL TO

NOISE RATIOS
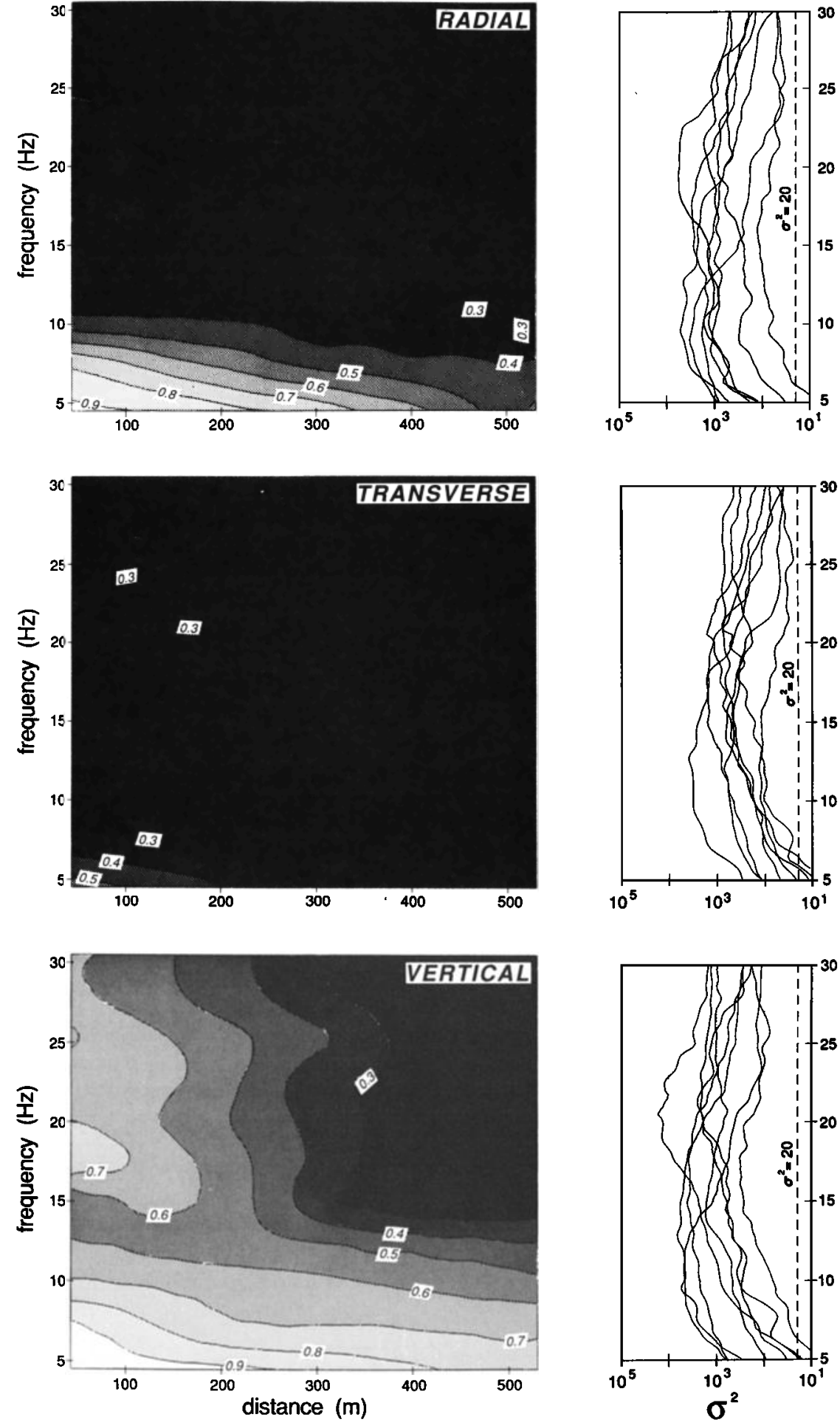

Fig. 8. Contour plots of average magnitude squared coherence for the components of the $P$ wave plotted against distance and frequency. Black signifies region where coherence has less than $95 \%$ confidence of being not equal to 0 . The right side plots show the average signal to noise ratio for all event for each phase. All data where the signal to noise ratio $\sigma^{2} \leq 20$ was not used for the contour plots.

Each of the contour plots shows, as expected, that $\gamma^{2}(f, d)$ increases as $f$ and $d$ decrease. The graph at the right side of each contour plots gives the signal-to-noise ratio for each event.

The most coherent signals are the vertical components of the compressional waves (which are nearly vertically incident).
These estimates have average values of $\gamma^{2}(f, d) \geq 0.6$ when $f=30$ $\mathrm{Hz}$ and $d=50 \mathrm{~m}$ and $\gamma^{2}(f, d) \geq 0.6$ when $f \leq 10 \mathrm{~Hz}$ and $d=500 \mathrm{~m}$. The least coherent signals are the transverse component of the compressional waves. Since the transverse component of the compressional wave is primarily scattered energy, the average 


\section{SHEAR WAVES}

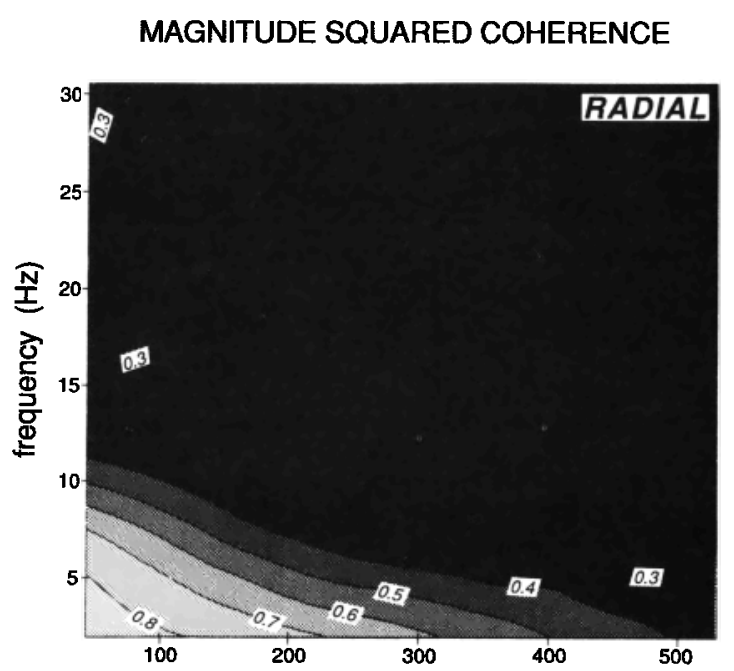

SIGNAL TO

NOISE RATIOS
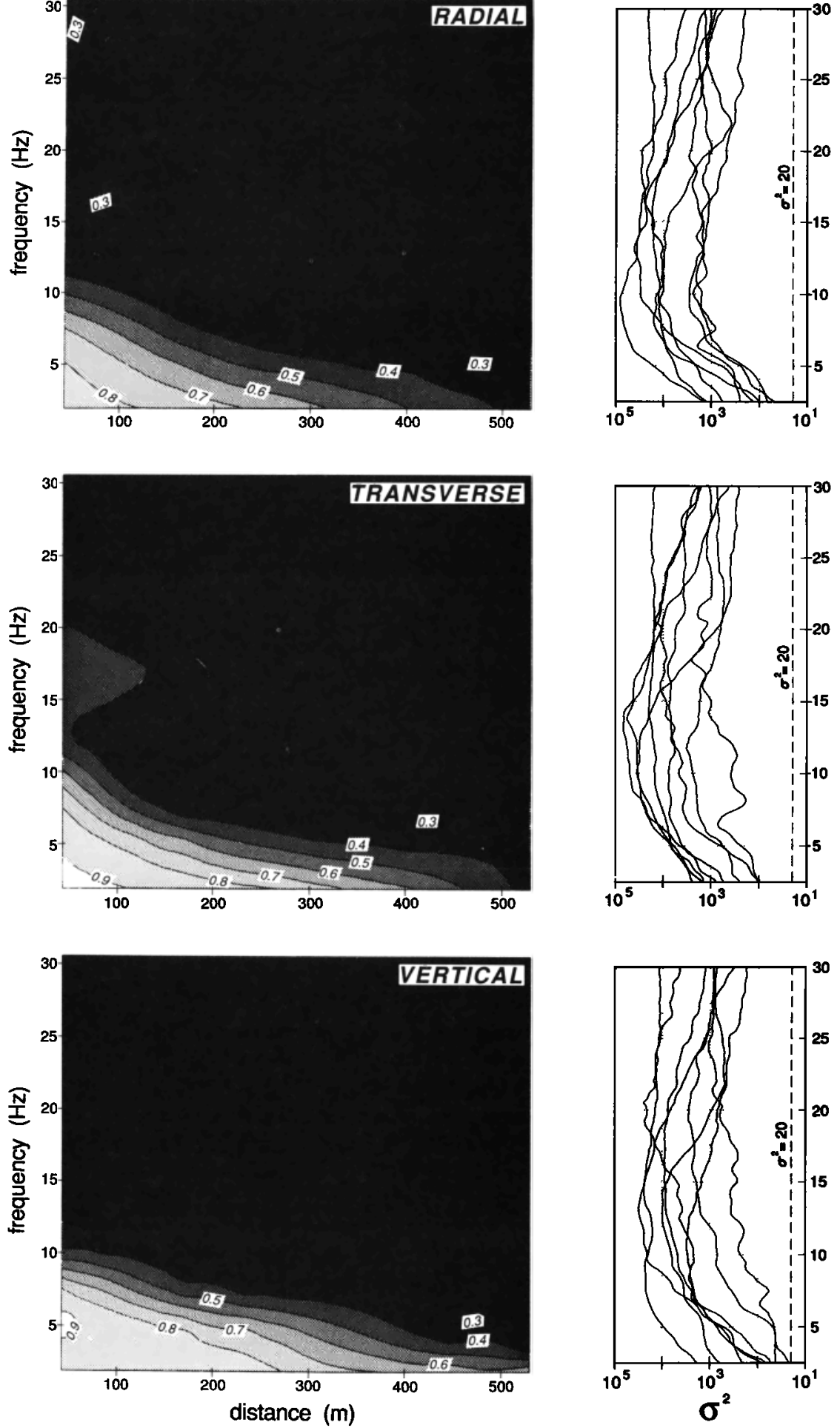

Fig. 9. Contour plots of average magnitude squared coherence for the components of the $S$ wave plotted against distance and frequency. Black signifies region where coherence has less than $95 \%$ confidence of being not equal to 0 . The right side plots show the average signal to noise ratio for all event for each phase. All data where the signal to noise ratio $\sigma^{2} \leq \mathbf{2 0}$ was not used for the contour plots.

measurements never exceed a value of $\gamma^{2}=0.5$ for the whole range of frequency and distance observed in this experiment. The radial component of the compressional wave should measure part of the direct wave as well as some scattered energy. The averaged coherence plot for this component does indeed have similar but lower values than the data from the vertical components.

The shear waves are also nearly vertically incident and should theoretically be separable into SH and SV polarized components. 
The SH polarization is observed on the transverse component, while the SV polarization is observed on the radial and vertical components. The highest averaged coherences for the $S$ waves are found on the vertical component. The next most coherent component of the $S$ waves is the transverse component, for which $\gamma^{2}(f, d)$ is significantly different from zero for $f \leq 20 \mathrm{~Hz}$ at $100 \mathrm{~m}$. The least coherent component of the shear waves is the radial component. It is important to note that at distances beyond $200 \mathrm{~m}$ and at frequencies greater than $10 \mathrm{~Hz}$, the average coherence is not significantly different from zero coherence for all components of the shear waves.

\section{BorEHOLE DATA}

Ideally, we would like to measure the coherence of the seismic wavefield in three dimensions. Unfortunately, not enough data exist from PFO to investigate the coherence properties as a function of depth. However, there are data from a set of borehole seismometers located at a site $25 \mathrm{~km}$ away. This site, $\mathrm{KNW}$, has very similar geological and topographical characteristics to those found at PFO [Fletcher et al., 1990]. The borehole seismometers are located at depths of $300(\mathrm{KN} 1)$ and $150(\mathrm{KN} 2) \mathrm{m}$. There is also a set of surface instruments $(\mathrm{KN} 3)$. The data consisted of compressional waves from 13 local earthquakes measured on the vertical components. The values for $\gamma^{2}(f)$, averaged for all events, are shown in Figure 10. Also plotted is the average $\gamma_{i j}^{2}(f)$ from the PFO array for station spacing $\Gamma_{\imath}=127 \mathrm{~m}$. At high frequencies the vertically separated sensors have greater coherence than surface sensors with the same spacing. The coherence between $\mathrm{KN} 1$ and $\mathrm{KN} 2$ is on average greater than that for $\mathrm{KN} 2-\mathrm{KN} 3$ which indicates that most of the signal distortion is occurring in the top $150 \mathrm{~m}$. Neither the source of the low coherence between $\mathrm{KN} 1$ and $\mathrm{KN} 2$ at $3 \mathrm{~Hz}$ nor the very pronounced scalloping effect in the $\mathrm{KN} 2-\mathrm{KN} 3$ coherence has been resolved yet. These effects are probably caused by the reflections of the compressional waves off the free surface.

\section{MODELING}

In this study the earthquakes have source dimensions of about $0.1-0.3 \mathrm{~km}$ and are small compared to the $15-40 \mathrm{~km}$ sourcereceiver distance. If the geology of the region is homogeneous, the seismic body wave would propagate effectively as a plane wave across the array. The body waves, however, have measurable variability in the power spectra and lose coherence when the station spacing is greater than $300 \mathrm{~m}$ for wavelengths shorter than $300-400 \mathrm{~m}$. These observations could be explained by various scattering mechanisms.

One widely used scattering model consists of a random velocity perturbation applied to a homogeneous velocity field. The perturbation field has correlation length $a$ and might have, for example, Gaussian, exponential, or a self-similar distribution [Chernov, 1960; Frankel and Clayton, 1986]. Studies of the velocity structure of the southern California region suggest that scale lengths of the order of tens of kilometers are present with velocity perturbations of less than $10 \%$ [Hearn and Clayton, 1986a, b]. Frankel and Clayton [1986] also concluded from their numerical studies that the Earth must have a correlation distance of over $10 \mathrm{~km}$.

If the scale length of $10 \mathrm{~km}$ is assumed, then this allows certain tests of the scattering model. Chernov [1960] found that the transverse autocorrelation for amplitude and phase approaches unity for station spacings $r_{t j}$ less than the scale length of the medium. Frankel and Clayton [1986] showed that the exponen-
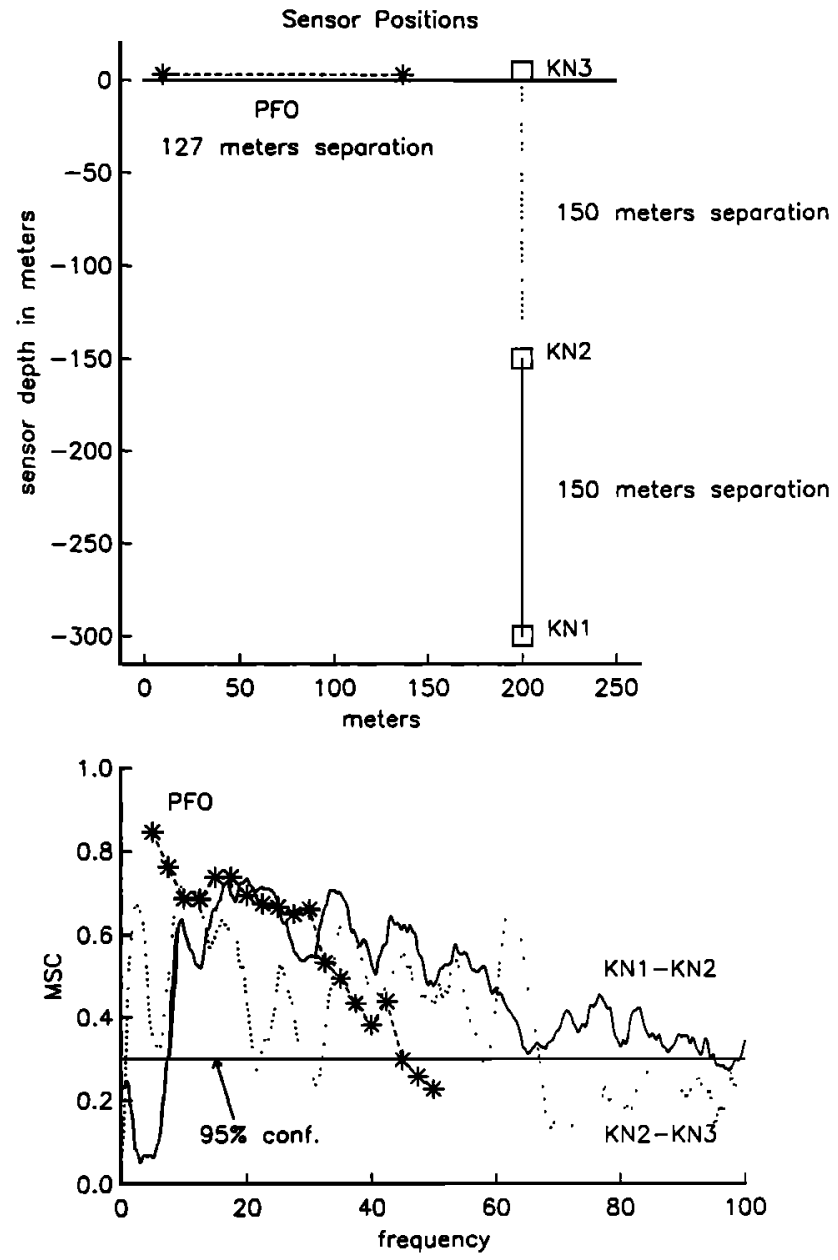

Fig. 10. Comparison of the averaged magnitude squared coherence estimates between sets of sensors spaced approximately $150 \mathrm{~m}$ apart. Three pairs of sensors are used, as shown in the top diagram, with two measurements in the vertical direction and one surface horizontal pair. The borehole sensors $\mathrm{KN1}$ and $\mathrm{KN} 2$ are placed at 300 and $150 \mathrm{~m}$ depth, respectively, while KN3 is located on the surface. The selected PFO data used station pairs which are $127 \mathrm{~m}$ apart. At least 10 events were used to form the average $\gamma^{2}$ for each pair of sensors. At frequencies above $30 \mathrm{~Hz}$ the coherence between KN1 and KN2 is much greater than the PFO surface values. The apparent holes in the $\gamma^{2}$ associated with KN1 and KN2 sensors is probably due to interference effects caused by the free surface reflection.

tial medium has similar properties to the Gaussian medium for $r_{i j} \ll a$. McLaughlin and Anderson [1987] showed that a Gaussian medium has the property that for fixed interstation distances $r_{i j} \ll a$ the $\gamma_{1 j}^{2}(f)$ will increase with frequency. These theoretical and numerical results indicate that for scale lengths of $a=10 \mathrm{~km}$ the seismic signals should be coherent over distances of less than $1 \mathrm{~km}$, which is clearly inconsistent with the data presented here.

Frankel and Clayton [1986] also studied a Von Karman medium, which has the property of being "self-similar" in the sense that the standard deviation of the medium remains constant over equal logarithmic intervals of wavenumber. They concluded that a self-similar random medium with long correlation length could explain the high-frequency content of the seismic coda. For a two-dimensional model with $a=10 \mathrm{~km}$ and a standard deviation of $5 \%$, their average band passed cross-correlation estimates decreased both as frequency and distance increased, similar to the results from the PFO small-aperture array. 
A set of synthetic seismograms was calculated for $\boldsymbol{P}$ wave propagating through a model with the same parameters as the twodimensional self-similar medium using the same program by Frankel and Clayton [1986]. Figure 11 displays the contours of coherence of $\boldsymbol{P}$ waves on the vertical component as functions of interstation spacing and frequency for this synthetic data. When compared to the coherence data from the small-aperture array (Figure 8), it is apparent that the synthetic seismograms are essentially coherent over $800 \mathrm{~m}$ distances. The self-similar medium with these parameters does not explain the array data. A much larger standard deviation $(\gg 5 \%)$ could possibly replicate the results from actual data, but this would be difficult to justify in the context of having both the earthquake source and the sensor location in the same granitic batholith.

Synthetic seismograms were also calculated for an exponential medium with $200 \mathrm{~m}$ correlation length and $10 \%$ standard deviation. The coherence of these signals (Figure 12) is less than the self-similar model but much higher than the small aperture array data, especially at station separations greater than $250 \mathrm{~m}$. It is interesting to note that the lowest coherence for this random medium occurs at $300 \mathrm{~m}$ and $17 \mathrm{~Hz}$. The minimum coherence distance of $300 \mathrm{~m}$ is similar to the correlation length, and a frequency of $17 \mathrm{~Hz}$ also has a wavelength of $-300 \mathrm{~m}$. As with the selfsimilar medium, a larger standard deviation would be necessary to replicate the array data.

\section{Discussion}

The spectral and coherence estimates presented are similar to measurements made at other locations. Tucker et al. [1984] compared spectra by calculating displacement amplitude spectral ratios between stations. They recorded earthquakes with different azimuths and angles of incidence in the frequency band $1-30 \mathrm{~Hz}$ and found that the spectral ratios vary by no more than a factor of 3 for their two surface hard rock sites. King and Tucker [1984] also calculated spectral ratios for their data with stations across a shallow valley filled with sediments overlying hard rock. In that experiment, amplifications as high as 10 were found between hard rock sites and sediment sites, while adjacent sites in the sediments

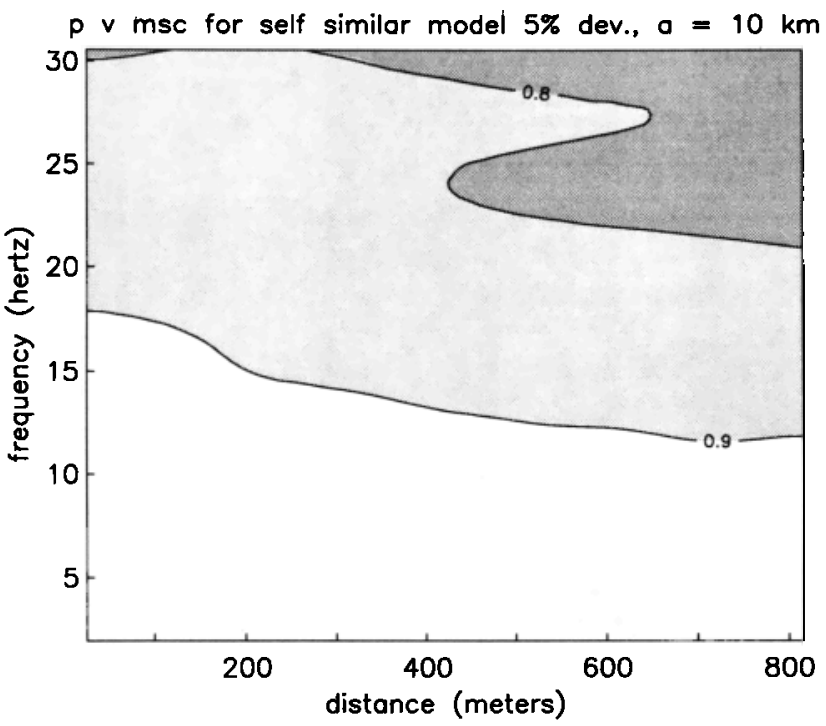

Fig. 11. Contour plots of average magnitude squared coherence for the vertical component of a $\boldsymbol{P}$ wave propagated through a self-similar random medium plotted against distance and frequency. Black signifies region where coherence has less than $95 \%$ confidence of being not equal to zero.

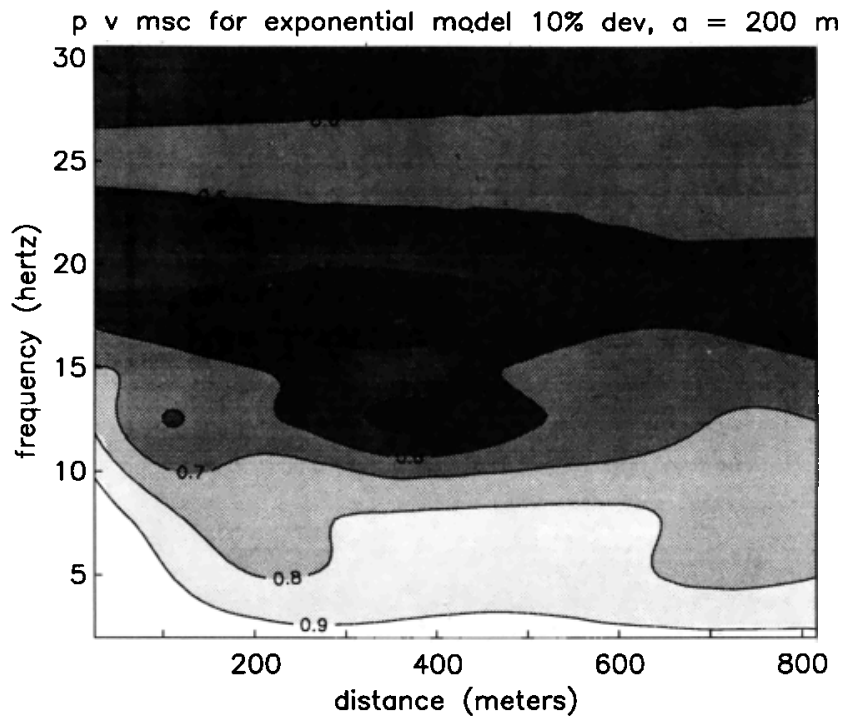

Fig. 12. Contour plots of average magnitude squared coherence for the vertical component of a $\boldsymbol{P}$ wave propagated through a exponential random medium plotted against distance and frequency. Black signifies region where coherence has less than $95 \%$ confidence of being not equal to zero.

with less than $100 \mathrm{~m}$ spacing showed spectral ratios of up to a factor of 5 .

To make a direct comparison between the prior results and the current data set, both average and maximum amplitude spectral ratios were calculated for the vertical components of the $P$ waves and the transverse components of the $S$ waves as shown in Figures 13 and 14. Each trace in these plots shows the spectral ratio as a function of frequency for one event. The average spectral ratios for this array can be as high as a factor of 2 , while the individual peak maximum values reach almost a factor of 7 . The station pair with the maximum spectral ratio for an event can be different for different frequencies. This is caused by the intermixing of the spectra which is shown in Figure $5 b$. Careful examination of all events show that the station pair with the maximum spectral ratio at a specific frequency changes from event to event. The spectral ratio also varies from event to event for each particular station pair. The average spectral ratios are comparable to the hard rock site results of Tucker et al. [1984], but the peak values meet or exceed the peak ratios which King and Tucker [1984] found for adjacent sediment sites. For reference, if this were a true stationary homogeneous process, then the expected $1 \%$ quantile is a ratio of approximately 2.7, using the Cochran Maximum $F$ statistic [Pearson and Hartley, 1969].

The data presented in this paper have the advantage of a good distribution of source azimuths so that the average properties of the site can be investigated. The $\gamma^{2}$ estimates have a high degree of variability as a function of frequency, superimposed on a general trend of decreasing $\gamma^{2}$, as the interstation spacing and the frequency increase; this agrees with the results of Menke et al. [1990] Examination of all $P$ wave $\gamma^{2}$ estimates on the vertical components shows that station pairs with $\gamma^{2}(f) \geq 0.8$ could be found for all $f \leq 45 \mathrm{~Hz}$, and $f \leq 20 \mathrm{~Hz}$ for transverse $S$ waves. Unlike the results of McLaughlin et al. [1983], the $\gamma^{2}$ estimates did not correlate with radial or transverse distance. For each event there might be a small apparent correlation, but this vanishes when averaged over all events. The $\gamma^{2}$ estimates also showed no correlation with azimuth or station pairs and are independent of the properties of the earthquake source focal mechanism when the data from all events are examined. 

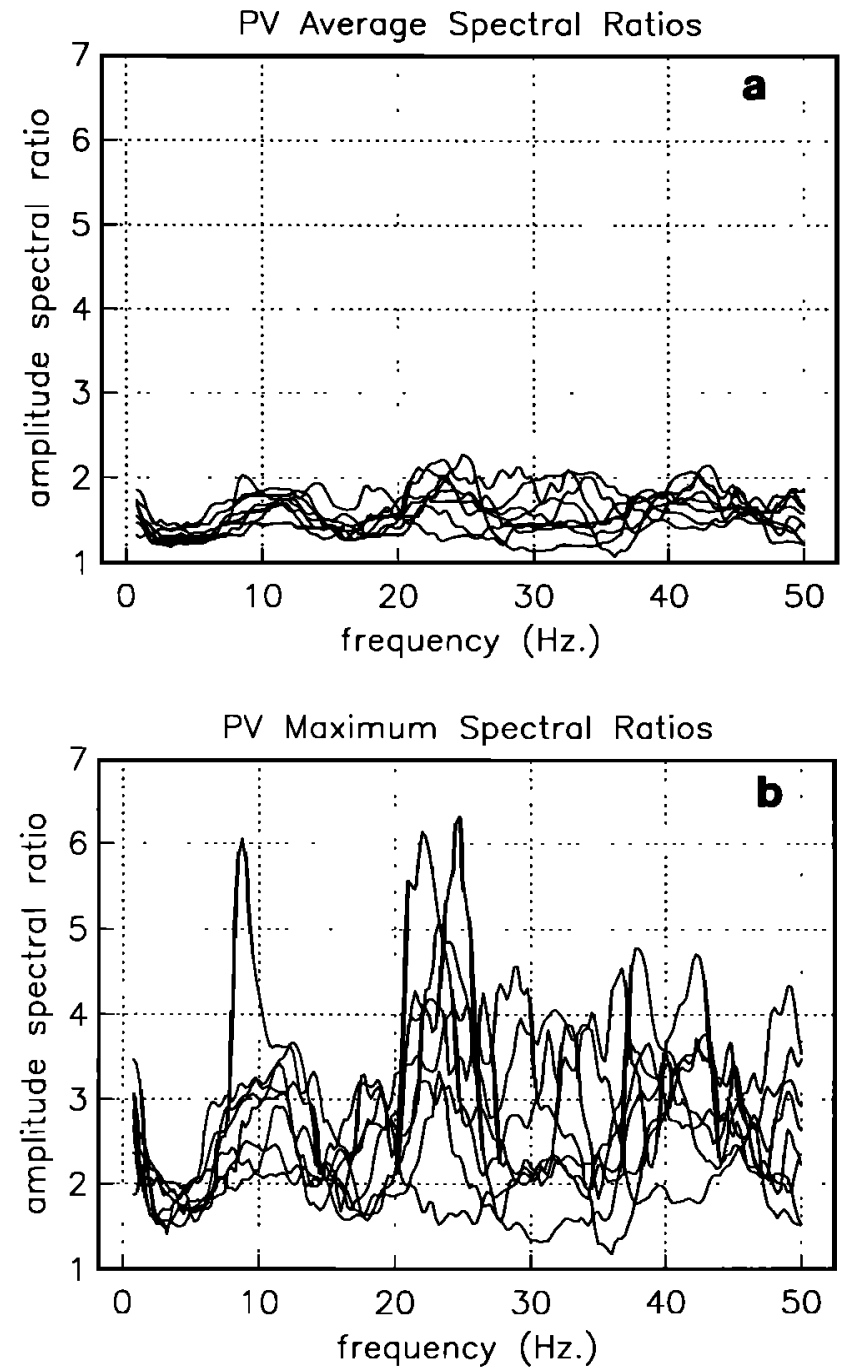

Fig. 13. (a) Average amplitude spectral ratios between all station pairs for each event on the $P$ wave, vertical component. (b) Maximum amplitude spectral ratio at each frequency between any station pair for each event on the $P$ wave, vertical component.

The only coherent $\left(\gamma^{2}>0.8\right)$ frequencies for both body wave phases are usually below the observed displacement amplitude corner frequencies $\left(f_{c}\right)$ of each respective phase. The measured corner frequencies at PFO for these events were between $11 \mathrm{~Hz}$ and $23 \mathrm{~Hz}$ for $P$ waves and between $6 \mathrm{~Hz}$ and $15 \mathrm{~Hz}$ for $S$ waves. The observation that the seismic waves are incoherent at the frequencies where $f_{c}$ is estimated for these events indicates that the comer frequencies might not be reliably determined and may be modified by local site effects. One possible explanation for low coherence at the comer frequencies is that the time windows used for analysis were too long and contained scattered energy after the initial coherent body wave pulse. Results from time windows of $0.5 \mathrm{~s}$ did not vary significantly from those calculated with $2.0-\mathrm{s}$ windows. However, it is possible to argue that since the comer frequency indicates a source duration of less than $0.1 \mathrm{~s}$, the window lengths for the coherence analysis should be equally short. In this experiment the estimates of coherence would then be calculated from two time series which contain a maximum of 20 data points each. The frequency resolution, at best, will be $10 \mathrm{~Hz}$ for spectra and coherence calculated from these time series. For analyzing time series with so few samples, a different or new set of techniques should be developed and applied.
The attempt to model the coherence results from PFO by propagating synthetic seismograms through media with random velocity variations did not yield satisfactory results. When correlation lengths and velocity perturbations which have been previously determined for southern California are used, the coherence estimates from these models are too high when compared to our data. It appears that with much larger random velocity variations $(\gg 10 \%$ ) over the whole volume of the crust, the results from PFO might be approximated. However, a model with such large velocity variations over the whole volume of the crust, while fitting the PFO data, would not be supported by other geophysical or geological data.

Another type of scattering model is an undulating interface separating the weathering layer from a homogeneous crystalline rock basement. All the measurements of the near-surface velocity structure at PFO have been single-point measurements. At present the lateral variability of the weathering layer is unknown but it is reasonable to expect that its depth could vary. Theoretical models of low-velocity materials overlying curved boundaries have been studied by Bravo et al. [1988] and Sánchez-Sesma et al. [1988] among others. These calculations show similar variations
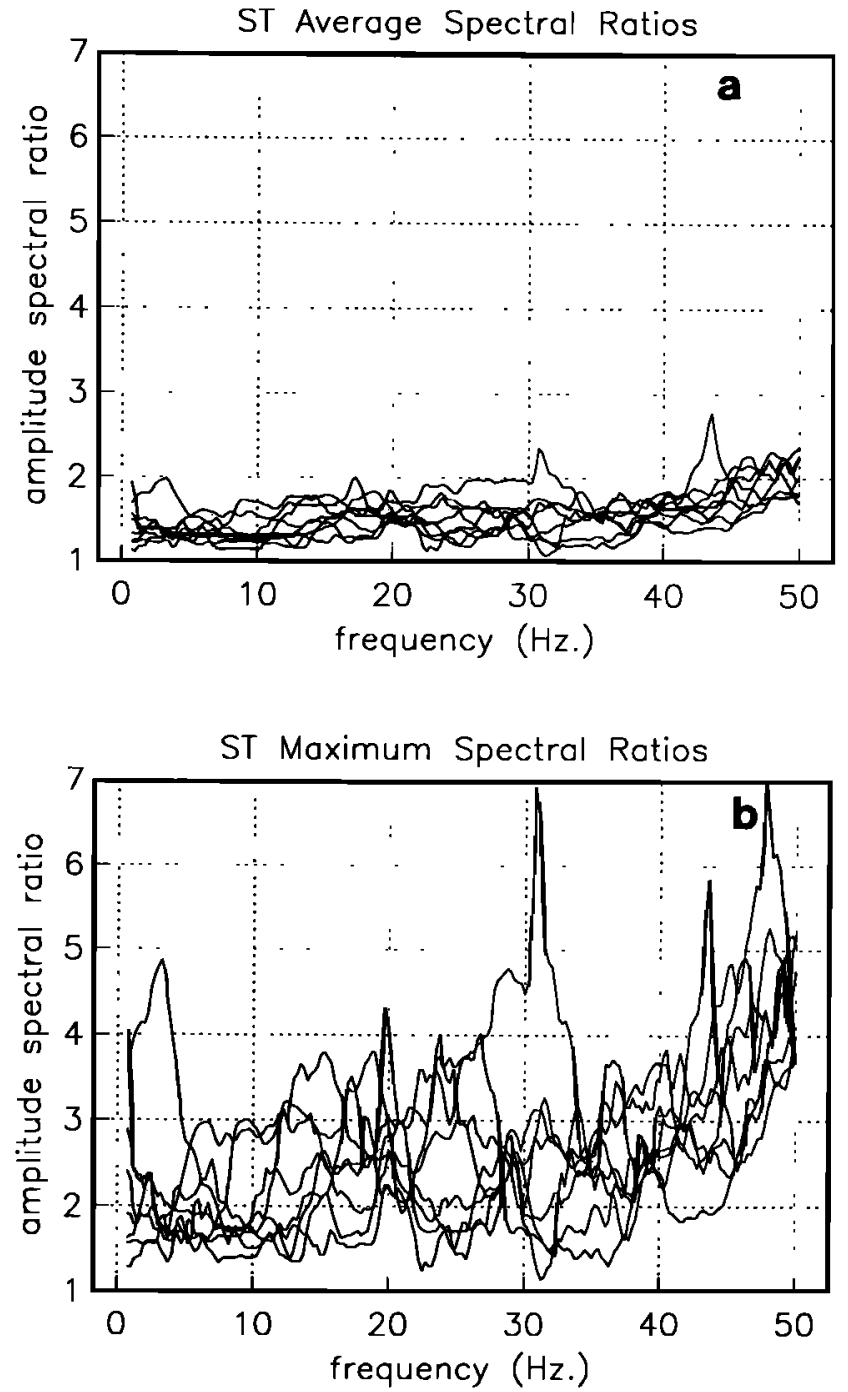

Fig. 14. (a) Average amplitude spectral ratios between all station pars for each event on the $S$ wave, vertical component. (b) Maximum amplitude spectral ratio at each frequency between any station pair for each event on the $S$ wave, vertical component. 
in amplitude, as functions of frequencies, similar to those observed in this experiment. Bravo et al. [1988] show examples of synthetic seismograms that display characteristics consistent with observed on this array. Using both parabolic and semicircular deposits over a more rigid basement, they show amplitude ratios as large as 16 between surface points at a given frequency. They demonstrated that the amplitude ratios measured between two points in their model are very dependent on the angle of incidence and on the frequency of the signal. Their results are consistent with those shown in Figure 14. A model in which there is an undulating depth of the weathering layer which causes most of the signal variations is perhaps the preferable interpretation for our data. The results from the KNW boreholes, showing higher coherence between two subsurface sensors than for equivalently spaced surface sensors, also fit this hypothesis.

\section{CONCLUSIONS}

During the operation of this small-aperture seismic array at Pinyon Flat, California, eight earthquakes were recorded. The site was chosen for its homogeneous granitic geology and its planar topography. The sensors and analog filters in the data loggers were all calibrated and had identical response functions. Power spectra were calculated for each body wave arrival. The spectra for each seismic phase and sensor component were log averaged together. A two standard deviation confidence limit was calculated, which is, on average, a multiplicative factor of 3 times the mean spectral level. Magnitude-squared coherences were calculated for all recordings at different sites. The magnitude-squared coherence estimates were highest for the $P$ wave arrivals on the vertical component and lowest for the $P$ wave recorded on the transverse component. Coherence for both the $P$ and $S$ waves decreases as frequency increases and distance increases.

The results here suggest that even for sites that appear to be optimal based on surficial features, the measured seismic wavefield can be distorted substantially over scale lengths of $500 \mathrm{~m}$. For sensors located within this distance, the general properties of the spectra are consistent, but the details are uncorrelated at higher frequencies. Synthetic seismograms were computed for velocity models with exponential and self similar distribution perturbations from which their coherence properties were calculated. Standard deviations $(-10 \%)$ are not sufficient for the random velocity distribution models to approximate the experimental results from the small-aperture array.

Even though the surface geology and topography are constant, a near-surface weathering layer with variable depth could cause this phenomenon. If so, a possible way to avoid this problem at this site is to place the sensors in boreholes. The coherence of signals from borehole sensors located $150 \mathrm{~m}$ apart vertically display higher average coherence than equally spaced sites located on the surface. To resolve whether a variable depth weathering layer is the source of the incoherence for the surface measurements at this location, data from closely spaced boreholes would have to be obtained and analyzed.

\section{APPENDIX: MULTIPLE WINDOW SPECTRUM ANALYSIS}

The multiple prolate window method is a the modem replacement for band-averaging methods whose advantages are summarized by Thomson [1982]. This appendix gives derivations for both the univariate and multivariate cases that differ slightly from the original forms of Thomson [1982].

Given a regular data sequence $x(n), n=1, \ldots N$, its discrete Fourier transform (DFT) is defined by

$$
y(f)=\sum_{n=0}^{N-1} e^{-i 2 \pi f(n-(N-1) / 2)} x(n)
$$

where time is written in centered form to keep with the notation of Slepian [1978] and frequency is a continuous variable on the principal domain $(-1 / 2,1 / 2]$.For a stationary process, the inverse Fourier transform associated with (A1) may be written in terms of the stochastic or Cramér representation

$$
x(n)=\int_{-1 / 2}^{1 / 2} e^{i 2 \pi v(n-(N-1) / 2)} d Z(v)
$$

where $d Z(f)$ is an orthogonal increment measure of the spectrum. This formulation is used to accommodate spectral lines; see Brillinger [1980] for a discussion. If the process has no line components, we have

$$
\begin{gathered}
\mathbf{E}[d Z(f)]=0 \\
\mathbf{E}\left[d Z(f) d Z^{*}(f)\right]=S(f) d f
\end{gathered}
$$

where $S(f)$ is the true power spectral density.

Combining (A1) with (A2) and recognizing the resulting sum as the Dirichlet kernel gives

$$
y(f)=\int_{-1 / 2}^{1 / 2} \frac{\sin N \pi(f-v)}{\sin \pi(f-v)} d Z(v)
$$

This is the fundamental equation of spectral analysis which describes a projection operation from the infinite stationary process represented by $d Z(f)$ onto the finite sample $y(f)$ using the Dirichlet kernel as a window through which the true spectrum is viewed. It should be remembered that it is the statistics of $d Z(f)$ that are of interest, not those of the DFT $y(f)$.

Formally, (A3) is a linear integral equation of the first kind and is solved approximately to give the multiple window method. The general approach given by Thomson [1982] is based on a weighted eigenfunction expansion of $d Z(f)$. The eigenfunctions of the Dirichlet kernel are the discrete prolate spheroidal wave functions $U_{k}(N, W ; f)$ described by Slepian [1978]. The parameter $W$ is a frequency to be chosen but typically $O\left(N^{-1}\right)$ which defines the inner or resolution bandwidth $2 W$. The eigenvalues $\lambda_{k}$ of the discrete prolate spheroidal wave functions (DPSW) give the fractional energy concentration of the DPSW in the inner band. The Fourier transforms of the DPSW are called discrete prolate spheroidal sequences (DPSS) and denoted by $\boldsymbol{v}_{n}^{(k)}$. It is assumed that the reader is familiar with both the standard notation given by Slepian [1978] and the properties of the DPSW and DPSS.

Neglecting mathematical rigor, the approximate solution $d \hat{Z}(f)$ to (A3) will be expanded in DPSW over the inner domain $(-W, W)$ using weight functions $y_{k}$ in the usual way to give

$$
d \hat{Z}\left(f ; f_{0)}=\sum_{k} b_{k} y_{k}\left(f_{0}\right) U_{k}\left(N, W ; f-f_{0}\right)\right.
$$

where the $\left\{b_{k}\right\}$ will be chosen to yield the correct spectrum for a white noise process, the solution is valid on $f_{0}-W \leq f \leq f_{0}+W$, and the upper limit to the sum will be specified later. For the moment, we are not weighting the terms of (A4) in a data adaptive manner to minimize bias. The Fourier-Bessel expansion coefficients are

$$
y_{k}\left(f_{0)}=\lambda_{k}{ }^{-1} \int_{-W}^{W} d s U_{k}(N, W ; s) y\left(s+f_{0}\right)\right.
$$

The normalization in (A5) is such that the true spectrum $S(f)$ will be obtained for a white noise process, where bias is not a con- 
sideration. To see this, substitute (A3) for $y(f)$ in (A5) to get

$$
y_{k}\left(f_{0}\right)=\int_{-1 / 2}^{1 / 2} U_{k}(f) d Z\left(f+f_{0}\right)
$$

and take the expected value of $\left|y_{k}(f)\right|^{2}$.

The $\left\{y_{k}\right\}$ are computed numerically using the DFT (A1) after windowing with the DPSS. To verify this, substitute (A1) into (A5), interchange the order of integration and summation, and substitute the complex conjugate of the relation between the DPSW and DPSS to get

$$
y_{k}\left(f_{0}\right)=\varepsilon_{k}{ }^{-1} \sum_{n=0}^{N-1} e^{-i 2 \pi f(n-(N-1) / 2)} v_{n}{ }^{(k)}(N, W) x(n)
$$

The expansion coefficient (A7) will be called the $k$ th eigentransform, while its square will be called the $k$ th eigenspectrum and is denoted by $\hat{S}_{k}(f)$. The latter is a direct estimate and is $\chi_{2}^{2}$ distributed.

From the bias properties of the prolate functions, there is little to be gained by using more than the first $K=2 N W$ DPSS as data windows. This determines the upper limit to the sum in (A4). A high-resolution spectral estimate follows by squaring (A4) and normalizing, implicitly invoking the ergodic hypothesis

$$
\hat{S}_{h}\left(f ; f_{0}\right)=\frac{1}{N}\left|\sum_{k=0}^{k-1} b_{k} y_{k}\left(f_{0}\right) U_{k}\left(N, W ; f-f_{0}\right)\right|^{2}
$$

Note that the high-resolution estimate has a free parameter, since it is valid both for $f_{0}-W \leq f \leq f_{0}+W$ and for $f-W \leq f_{0} \leq f+W$. This property will be exploited later. It is also a statistically inconsistent estimate, since its variance does not decrease as $N$ increases, and is $\chi_{2}^{2}$ distributed. To obtain statistical consistency, we take its average over the inner bandwidth to get the averaged spectrum $\hat{S}\left(f_{0}\right)$

$$
\begin{aligned}
\hat{S}\left(f_{0}\right) & =\frac{1}{2 W} \int_{f_{0}-W}^{f_{0}+W} d f \hat{S}_{h}\left(f ; f_{0}\right) \\
& =\frac{1}{K} \sum_{k=0}^{K-1} \lambda_{k} b_{k}{ }^{2}\left|y_{k}\left(f_{0}\right)\right|^{2}
\end{aligned}
$$

where the orthogonality properties of the DPSW are exploited to perform the integration. The coefficients $b_{k}$ follow from the requirement of correct normalization for a white noise process. The eigenspectra are already normalized so that $\mathbf{E}\left[\hat{S}_{k}(f)\right]=S(f)$, hence $b_{k}=\sqrt{\lambda_{k}^{-1}}$ to yield that result for the averaged spectrum. This spectral estimate has $2 K$ degrees of freedom, since it is the average of $K$ eigenspectra with 2 degrees of freedom apiece.

Due to the poor bias properties of higher-order eigenspectra when the spectrum is (relatively) small, the straight averaging used in (A9) is not recommended. Instead, we may generalize (A8) to

$$
\hat{S}_{h}\left(f ; f_{0}\right)=\frac{A}{N} \frac{\left|\sum_{k=0}^{K-1} d_{k}(f) y_{k}\left(f_{0}\right) U_{k}\left(f-f_{0}\right)\right|^{2}}{\sum_{k=0}^{K-1}\left|d_{k}(f)\right|^{2}}
$$

where the weights $d_{k}(f)$ are computed adaptively from the data in a manner which minimizes the broadband bias outside of the inner band. The scale factor $A$ will then be chosen to yield the correct result for a white noise process in the usual way. Considering the definition of the averaged spectrum (A9) and assuming that the spectrum does not change too rapidly in the inner band about $f_{0}$, so that $d_{k}(f) \approx d_{k}\left(f_{0}\right)$, we obtain

$$
\hat{S}\left(f_{0}\right)=\frac{A}{K} \frac{\sum_{k=0}^{K-1} \lambda_{k} d_{k}^{2}\left(f_{0}\right)\left|y_{k}\left(f_{0}\right)\right|^{2}}{\sum_{k=0}^{K-1} d_{k}^{2}\left(f_{0)}\right.}
$$

The weights are computed by minimizing the squared difference between the true and estimated Fourier-Bessel coefficients. The latter are given by (A6), while the true Fourier-Bessel coefficients (which cannot be observed) are given by the definition as

$$
d Z_{k}\left(f_{0}\right)=\sqrt{\lambda_{k}^{-1}} \int_{-w}^{W} U_{k}(v) d Z\left(v+f_{0}\right)
$$

The normalization of (A12) is again correct for white noise, as can be verified by taking its expected value. The weights will be chosen to minimize

$$
\varepsilon_{k}{ }^{2}=\left|d Z_{k}(f)-d_{k}(f) y_{k}(f)\right|^{2}
$$

at each frequency $f$. Using (A6) and (A12), this may be reexpressed as

$$
\begin{aligned}
\varepsilon_{k}^{2}=\mid\left[\sqrt{\lambda_{k}^{-1}}-d_{k}(f)\right] \int_{-W}^{W} U_{k}(s) d Z(s+f) & \\
& -\left.d_{k}(f) \underbrace{1 / 2}_{-1 / 2} U_{k}(s) d Z(s+f)\right|^{2}
\end{aligned}
$$

where the cut integral excludes the interior domain $(-W, W)$. This may be expanded, and since the cross terms vanish, the expected values are

$$
\begin{aligned}
& \mathbf{E}\left[\left|\int_{W}^{W} U_{k}(s) d Z(s+f)\right|^{2}\right]=\lambda_{k} S(f) d f \\
& \mathbf{E}\left[\left.||_{-1 / 2}^{1 / 2} U_{k}(s) d Z(s+f)\right|^{2}\right]=B_{k}(f) d f \leq \sigma^{2}\left(1-\lambda_{k}\right) d f
\end{aligned}
$$

where $\sigma^{2}$ is the data sample variance. The second term is the broadband bias (by definition), and the bound may be obtained with Schwartz's inequality. It is an equality for a white noise process, suggesting the use of prewhitening before data processing. The squared error reduces to

$$
\varepsilon_{k}^{2} \approx\left[\sqrt{\lambda_{k}^{-1}}-d_{k}(f)\right]^{2} \lambda_{k} S(f)+d_{k}^{2}(f) B_{k}(f)
$$

Minimizing this in the usual way yields the approximate weights

$$
d_{k}(f)=\frac{\sqrt{\lambda_{k}} S(f)}{\lambda_{k} S(f)+B_{k}(f)}
$$

Note that $d_{k}(f)=b_{k}$ in the absence of broadband bias $\left(B_{k}(f)=0\right)$, reducing (A10) to the earlier result (A8) if

$$
A=\sum_{k=0}^{K-1} \lambda_{k}{ }^{-1}
$$

As the broadband bias rises, the weights decrease, downweighting portions of the eigenspectra which are dominated by bias. To compute the weights, the true spectra in (A13) are replaced by the estimate (A11), yielding the recursive equation

$$
\sum_{k=0}^{K-1} \frac{\lambda_{k}\left(\hat{S}(f)-\lambda_{k} \frac{A}{K} \hat{S}_{k}(f)\right)}{\left(\lambda_{k} \hat{S}(f)+\hat{B}_{k}(f)\right)^{2}}=0
$$


This may be solved iteratively using standard methods, and the weights are then computed using (A13). An estimate of the equivalent degrees of freedom is given by

$$
v(f)=2 \sum_{k=0}^{K-1} \lambda_{k} d_{k}^{2}(f)
$$

Note that this yields the maximum possible value $2 K$ only in the absence of bias and will generally be smaller, especially at low points in the spectrum. A useful indicator of the extent of bias in the estimate can be obtained by averaging (A15) over frequency. If the average is substantially lower than $2 K$, then either the choice of interior domain frequency $W$ should be increased or the data should be prewhitened more carefully.

The spectral representation given by (A1) and (A2) admits the presence of harmonic lines quite conveniently, and it is possible to detect and remove such components from the stochastic part of the spectrum. This process is called reshaping; see Thomson [1982] for details.

These procedures may easily be extended to the bivariate and multivariate cases. Consider a set of $p$ time series $x_{j}(n), n=0, \ldots, N-1, j=1, \ldots, p$. We first compute the univariate spectrum and adaptive weights using the methods already described. This includes reshaping of significant spectral lines, as the coherences and transfer functions are ordinarily defined only for the stochastic portion of the spectrum. The averaged power of the $j$ th series analogous to (A11) is given by

$$
\hat{S}_{J j}(\omega)=\frac{A}{K} \sum_{i=0}^{K-1} \lambda_{i}\left(e_{i}^{J}(\omega)\right)^{2}\left|y_{i}^{J}(\omega)\right|^{2}
$$

where

$$
e_{i}^{J}(\omega)=d_{i}^{J}(\omega) /\left[\sum_{i=0}^{K-1}\left(d_{i}^{\prime}(\omega)\right)^{2}\right]^{\frac{1}{2}}
$$

is a normalized adaptive weight. The subscript on the terms in (A16) refers to the prolate order, while the superscript refers to the series number. An obvious extension to the cross spectrum is

$$
\hat{S}_{J m}(\omega)=\frac{A}{K} \sum_{i=0}^{K-1} \lambda_{l} e_{i}^{\prime}(\omega) e_{i}^{m}(\omega)\left(y_{i}^{\prime}(\omega)\right)^{*} y_{\imath}^{m}(\omega)
$$

using the adaptive weights from the respective autopowers. The simple coherence for the case $p=2$ follows immediately as

$$
\gamma_{j m}^{2}=\frac{\left|\hat{S}_{j m}(\omega)\right|^{2}}{\left|\hat{S}_{j J}(\omega)\right|\left|\hat{S}_{m m}(\omega)\right|}
$$

while the corresponding phase is just that of (A17). Similar expressions may be derived for the multivariate transfer functions, multiple and partial coherences, and empirical orthogonal functions (frequency domain principal components).

Acknowledgments. We greatly appreciate the helpful comments given by David Thomson, Tom Hanks, and Frank L. Vernon, Jr. We would like to thank Art Frankel for calculating the synthetic seismograms used in this paper. This work would not have been possible without the cooperation of Frank Wyatt and Leo Weuve and the shelter provided by John Reuyal and Allan and Becky Ota. This work was supported by U. S. Geological Survey contract 14-08-0001-A0614.

\section{REFERENCES}

Bravo, M. A., F. J. Sánchez-Sesma, and F. J. Chánez-Garci," Ground motion on stratified alluvial deposits for incident SH waves, Bull. Seismol. Soc. Am., 78, 436-450, 1988.
Brillinger, D. R., Time Series, Holden-Day, Oakland, Calif., 1980

Chernov, L., Wave propagation in a random medium, McGraw-Hill, New York, 1960.

Dibblee, T. W., Geology of the San Jacinto Mountains and adjacent areas, in Geology of the San Jacinto Mountains, pp. 1-47, South Coast Geological Society, Costa Mesa, Calif., 1981.

Efron, B., The Jackknife, The Bootstrap, and Other Resampling Plans, CBMS-NSF Reg. Conf Ser. in Appl. Math., Vol. 38, Society for Industrial and Applied Mathematics, Philadelphia, Pa., 1982.

Fletcher, J., L. Haar, T. Hanks, L. Baker, F. L. Vernon III, J. Berger, and J. N. Brune, The digital array at Anza, J. Geophys. Res., 92, 369-382, 1987.

Fletcher, J. B., T. Fumal, H. Liu, and R Porcella, Near-surface velocities and attenuation at two bore-holes near Anza, CA from logging data, Bull. Seismol. Soc. Am., 80, 807-831, 1990.

Frankel, A., and R. W. Clayton, Fintte difference simulations of seismic scattering: Implications for the propagation of short-period seismic waves in the crust and models of crustal heterogenetty, J. Geophys Res., 91, 6465-6489, 1986.

Geli, L., P. Bard, and B. Jullien, The effect of topography on earthquake motion, Bull. Seismol. Soc. Am., 78, 42-63, 1988.

Given, D. D., L. K. Hutton, and L. M. Jones, The Southern California Network Bulletin July-December 1986, U.S. Geol. Surv. Open File Rep. 87-844, 1987.

Hearn, T. M., and R. W. Clayton, Lateral velocity variations in Southern California, I, Results for the upper crust from $P_{g}$, Bull. Selsmol. Soc. Am., 76, 495-510, 1986 .

Hearn, T. M., and R. W. Clayton, Lateral velocity variations in Southern California, II. Results for the lower crust from $P_{n}$, Bull. Seismol. Soc Am., 76, 511-520, $1986 b$.

Jenkins, G. M., and D. G. Watts, Spectral Analysls and Its Applications, Holden-Day, Oakland, Calif., 1968.

King, J. L., and B. E. Tucker, Observed variations of earthquake motion across a sediment-filled valley, Bull. Seismol. Soc. Am., 74, 153-165, 1984.

Lanzerotti, L. J., D. J. Thomson, A. Meloni, L. V. Medford, and C. G. Maclennan, Electromagnetic study of the Atlantic continental margin using a section of a transatlantic cable, $J$ Geophys. Res., 91, $7417-7427,1986$

Lindberg, C., and J. Park, Multiple-taper spectral analysis of terrestrial free oscillations, Part II, Geophys. J. R. Astron. Soc., 91, 795-836, 1987.

McLaughlin, K. L., and L. M. Anderson, Stochastic dispersion of short period $P$ waves due to scattering and multipathing, Geophys $J . R$. Astron Soc., 89, 933-963, 1987.

McLaughin, K. L., L. R. Johnson, and T. V. McEvilly, Two-dimensional array measurements of near-source ground accelerations, Bull. Seismol. Soc. Am., 73, 349-375, 1983.

Menke, W., A. L. Lerner-Lam, B. Dubendorff, and J. Pacheco, Polarization and coherence of 5 to $30 \mathrm{~Hz}$ seismic wave fields at a hard-rock site and their relevance to velocity heterogenieties in the crust, Bull. Seismol. Soc Am., 90, 430-449, 1990.

Miller, R. G., Jackknifing variances, Ann. Math. Stat., 39, 567-582, 1968.

Miller, R. G., The jackknife - A review, Biometrika, 61, 1-15, 1974

Norris, R., L. M. Jones, and L. K. Hutton, The Southern California Network Bulletin July 1 through December 31, 1985, U.S. Geol. Surv. Open File Rep. 86-337, 1986.

Parcel, R. F., Structure and petrology of the Santa Rosa shear zone in the Piñon Flat area, in Geology of the San Jacinto Mountains, pp. 139-150, South Coast Geological Society, Costa Mersa, Calif., 1981.

Park, J., C. R. Lindberg, and F. L. Vemon III, Multitaper spectral analysis of high-frequency seismograms, $J$. Geophys. Res, 92, 12,675-12,684, 1987.

Pearson, E. S., and H. O. Hartley, Biometrika Tables for Statisticıans, 3rd ed., Cambridge University Press, New York, 1969.

Sánchez-Sesma, F. J., F. J. Chávez-Garciá, M. A. Bravo, Seismic response of a class of alluvial valleys for incident $\mathrm{SH}$ waves, Bull. Seismol. Soc. Am., 78, 83-95, 1988.

Slepian, D., Prolate spheroidal wave functions, Fourier analysis, and uncertainty-V: The discrete case, Bell Syst.Tech.J.,57, 1371-1429, 1978.

Thomson, D. J., Spectrum estimation and harmonic analysis, Proc. IEEE, $70,1055-1096,1982$.

Tucker, B. E., and J. L. King, Dependence of sediment-filled valley response on input amplitude and valley properties, Bull. Seismol. Soc. Am., 74, 153-165, 1984.

Tucker, B. E., J. L. King, D. Hatzfeld, and I. L. Nersesov, Observations of hard-rock site effects, Bull. Seismol. Soc. Am., 74, 121-136, 1984. 
Tukey, J., Bias and confidence in not quite large samples, abstract, Ann Math. Stat., 29, 614, 1958.

Wyatt, F., Displacements of surface movements, J. Geophys. Res., 87, 979-989, 1982.

Wyatt, F., and J. Berger, Investigations of tilt measurements using shallow borehole tiltmeters, J. Geophys. Res., 85, 4351-4362, 1980.

L. Carroll, J. Fletcher, and E. Sembera, U.S. Geological Society, Office of Earthquakes, Volcanoes and Engineering, Menlo Park, CA 94025.
A. Chave, AT\&T Bell Laboratories, 1E444, 600 Mountain Avenue, Murray Hill, NJ 07974.

F. L. Vernon, Institute of Geophysics and Planetary Physics, A-025, University of California, San Diego, La Jolla, CA 92093.

\begin{abstract}
(Received March 19, 1990;
revised October 19, 1990;

accepted November 13, 1990.)
\end{abstract}

